\title{
CAMBIO ESTRATÉGICO PARA ENTORNOS TURBULENTOS
}

\author{
HUGO ALBERTO RIVERA RODRIGUEZ* \\ UNIVERSIDAD DEL ROSARIO, BOGOTÁ
}

Recibido/Received/Recebido: 03/03/2010 - Aceptado/ Accepted/Aprovado: 16/05/2010

\begin{abstract}
Resumen
En los últimos años, las empresas se han visto enfrentadas a un entorno turbulento, caracterizado principalmente por presentar incertidumbre, dinamismo y complejidad. La incertidumbre se hace evidente al momento de tomar decisiones; sin lograr tener certeza sobre lo que puede pasarle a la empresa; el dinamismo se logra observar por el cambio permanente en las necesidades de los clientes y surgimiento de nuevos productos, que origina incremento de la rivalidad por precio; y la complejidad puede identificarse en el surgimiento de nuevos actores con los que se debe interactuar para llevar a cabo una transacción. La academia de la administración ha buscado mecanismos para enfrentarse a la turbulencia. En el presente documento se presenta una revisión teórica del concepto de turbulencia, haciendo énfasis en la forma como debe enfrentarse el fenómeno mediante el cambio estratégico.
\end{abstract}

Palabras clave: entorno turbulento, cambio estratégico, incertidumbre, dinamismo, complejidad

\section{STRATEGIC CHANGE FOR TURBULENT ENVIRONMENTS}

\begin{abstract}
In the last years, companies have faced a turbulent environment, mainly characterized by uncertainty, dynamism and complexity. Uncertainty is evident at decision making moment; without certainty about what can happen to the company; dynamism is observed by permanent change of customer needs and emergence of new actors to interact with, in order to make a transaction. Business administration academy has looked for mechanisms to face this turbulence. This paper presents a theoretical review of turbulence concept, emphasizing on how this phenomenon must be faced through a strategic change.
\end{abstract}

Keywords: strategy, turbulent environment, uncertainty, dynamism, complexity

\section{MUDANÇA ESTRATÉGICA PARA AMBIENTES AGITADOS}

\section{Resumo}

Nos últimos anos, as empresas têm enfrentado um ambiente turbulento, caracterizado principalmente por a incerteza, o dinamismo e a complexidade. A incerteza ocorre quando se tomam decisões sem ter certeza do que pode acontecer com a empresa; o dinamismo se observa na mudança constante das necessidades do cliente e no surgimento de novos produtos, o que causa aumento na concorrência de preço; a complexidade pode identificar-se no surgimento de novos atores que intervêm para realizar uma transação. A academia da administração tem buscado meca-

Profesor principal de la Universidad del Rosario. El documento se encuentra asociado a la línea de investigación en estrategia, perteneciente al Grupo de investigación en Perdurabilidad empresarial. Correo electrónico: hugo.rivera@urosario.edu.co 
nismos para lidar com a turbulência. Neste artigo revisa-se teoricamente o conceito de turbulência, enfatizando a maneira de enfrentar o fenômeno mediante uma mudança estratégica.

Palavras chave: Ambiente agitado, mudança estratégica, incerteza, dinamismo, complexidade

Rivera, H. (2010). Cambio estratégico para entornos turbulentos. En: Revista de la Facultad de Ciencias Económicas de la Universidad Militar Nueva Granada. rev.fac.cienc.econ, XVIII (1)

JEL: M190

\section{Introducción}

El estudio de la turbulencia del entorno ha sido tema de investigación de la academia de la administración desde mediados del siglo XX. Sin embargo, no existe una puesta en común en aspectos como el concepto, las consecuencias, y la forma de enfrentar el fenómeno. Este documento que se encuentra dividido en dos secciones incluye una revisión de los principales tópicos de investigación sobre turbulencia. En la primera sección se define el concepto de turbulencia, haciendo énfasis en la dificultad para llegar a una única definición como resultado de la existencia de términos similares, sin límites claramente definidos, luego se presentan los factores generadores de turbulencia; y se hace una síntesis de los hallazgos encontrados sobre el impacto (positivo y negativo) de la turbulencia. A continuación se hace una revisión de los mecanismos utilizados en investigaciones previas para medir la turbulencia, para concluir la sección con las principales alternativas teóricas propuestas para enfrentar la turbulencia; En esta parte se encuentra que el cambio estratégico es la vía para enfrentar la turbulencia evitando una erosión en el desempeño de las empresas; por ello la segunda sección del documento se centra en la revisión del concepto de cambio estratégico, tema abordado por la literatura de administración desde mediados del siglo XX.

\section{El concepto de turbulencia}

Para la Real Academia Española, turbulencia significa cualidad de turbio, confuso, desordenado, al- borotado o perturbado. El Diccionario Merrian Webster define turbulencia como: gran conmoción o agitación. Los primeros autores que utilizaron el concepto de turbulencia de una manera precisa en el management fueron Emery y Trist (1965), quienes al estudiar el entorno propusieron cuatro tipos diferentes, donde el punto culminante sería un entorno caracterizado por el dinamismo y la incertidumbre al cual llamaron turbulento. Para ellos turbulencia es la inestabilidad o tasa de cambio subyacente en el ambiente de las organizaciones; es una situación donde los recursos y restricciones cambian constantemente, obligando a las empresas a reaccionar. Años después, Terreberry (1968) indicó que la turbulencia del entorno se caracteriza por una tasa acelerada y compleja de las interacciones que presenta la empresa, excediendo su capacidad para predecir y controlar las consecuencias de sus acciones.

Con posterioridad a los trabajos anteriores, las definiciones incorporan las causas del fenómeno. Para Galbraith (1973) la turbulencia se origina por el crecimiento económico, por avances científicos, y por sistemas de comunicaciones modernos. Para Khandwalla (1976/1977) la turbulencia se caracteriza por los cambios rápidos e imprevisibles en muchos aspectos del entorno. Pero fue Ansoff (1979) ${ }^{1}$ quien popularizó la noción de turbulencia, al manifestar que es la razón de ser del management, y que estaba en el líder gestionar las sorpresas y las discontinuidades propias de esta condición. Aunque esta reflexión teórica sirvió de plataforma para popularizar el concepto, estaba más enfocada al aná-

1 Ansoff (1990 y 1992) propuso diferentes niveles de turbulencia, indicó que estos niveles están caracterizados en función de la complejidad de los eventos del entorno, el conocimiento de la sucesión de acontecimientos, la rápida evolución de estos eventos y la visibilidad de estos futuros eventos. 
lisis de las responsabilidades del líder que al estudio del fenómeno como tal.

En el mismo año, Aldrich (1979) propuso a la turbulencia como una dimensión del entorno, definiéndola como el grado de interconexión entre los elementos que lo conforman. Ya en los años ochenta surgen nuevas definiciones, la mayoría de ellas se basan en las ideas ya expresadas en los años sesenta y setenta. Para Trist (1980), las organizaciones que se encuentran en un entorno turbulento actúan de manera independiente en diversas direcciones generando consecuencias negativas en el entorno que comparten. Por su parte Dess \& Beard (1984) y Bourgeois \& Eisenhardt (1988), manifestaron que un entorno turbulento presenta un alto grado de cambios esporádicos generadores de incertidumbre y dificultad en la predicción. Cameron, Kim \& Whetten (1987) establecieron que un entorno turbulento es aquel donde los cambios son significativos, rápidos y discontinuos.

Lo más revolucionario en materia de definiciones fue el concepto de hiperturbulencia, desarrollado por McCann \& Selski (1984) y D'Aveni (1994), y utilizado posteriormente por Bettis \& Hitt, (1995) y Brown \& Eisenhardt, (1998). En esta situación las demandas del entorno exceden la capacidades de adaptación de las empresas que intervienen en él y la estabilidad se encuentra constantemente amenazada por nuevos productos, nuevas tecnologías y nuevos competidores; obligando a las empresas a orientarse a la obtención de ventajas competitivas temporales (D’Aveni, 2004). El elemento diferenciador de esta propuesta se encuentra en la recomendación por generar ventajas transitorias como mecanismo de enfrentar el fenómeno.

A comienzos de la década de los noventa, Waterhouse (1992) diría que no es más que un estado, que se logra cuando existe un alto grado de complejidad e incertidumbre, acompañado de un bajo nivel de predicción, autonomía y control. En esta definición ya se incorporan dimensiones, además de evidenciar fallas internas en las empresas.

Glazer \& Weiss (1993) indican que la turbulencia se explica en los altos niveles de cambios en diferen- tes periodos en los factores del entorno. Mintzberg (1994) mostraría que la turbulencia no es algo malo, él indicó que aun cuando en un estado de turbulencia surgen problemas y cambios permanentes, no se puede hablar de él como un desorden violento, ya que la respuesta de las empresas da como resultado cambios en competencia y en la tecnología.

Sadler (1996) indicó que un entorno turbulento es aquel que presenta cambios frecuentes que ocurren en forma rápida y simultánea. García, Dolan \& Navarro (1999) lo definieron como un mercado en el que el choque de tendencias contradictorias o contrapuestas dificulta la toma de decisiones para la empresa, dada la incertidumbre existente.

En la presente década se encuentran definiciones que recogen lo ya encontrado anteriormente. Para Gueguen (2001) la turbulencia es una cadena de acontecimientos más o menos espaciados en el tiempo, más o menos favorables, pero impredecibles en cuanto a su magnitud, que son suficientemente nuevos para causar un impacto en las organizaciones, que son percibidos por sus miembros, generando una reconsideración en la estrategia de la empresa. Dante (2002) definió entorno turbulento como aquel en el que se producen una sucesión de cambios abruptos e imprevisibles que impactan desestabilizando el sistema en el que funciona la empresa, dificultando su capacidad de adaptación. Para Calantone, Garcia \& Droge (2003) es aquel en el que se presentan de manera frecuente $e$ impredecible cambios en el mercado y la tecnología que afecta el desarrollo de nuevos productos. Caldart \& Ricart (2006) indican que la turbulencia es una consecuencia de la dificultad que tienen muchas industrias para alcanzar una visión congruente de los conflictos externos con los que se enfrentan.

Recientemente Buganza, Del'Era \& Verganti (2009) argumentaron que la turbulencia indica el nivel de variabilidad del estado del entorno, el cual depende de la dinámica y la complejidad del mismo. Pocos cambios, o cambios graduales en condiciones de baja complejidad implican baja turbulencia; en cambio, modificaciones radicales y alta complejidad se reflejan en alta turbulencia. 
Como puede observarse, existe una confusión en la literatura sobre lo que es la turbulencia. La razón, de acuerdo con Woodward (1982) y posteriormente McCann \& Selski (1984), estaría en que no puede definirse turbulencia porque es una metáfora; la turbulencia para ellos es una condición relativa por la que atraviesan las firmas. Para Gueguen (2001), la falta de claridad conceptual se debe a que el concepto de entorno es multidimensional y los límites de estas dimensiones no están claramente definidos, llevando a que la sustitución de conceptos suplante la complementariedad de los mismos

\subsection{Conceptos similares}

En la revisión de literatura se encuentran rótulos con definiciones similares a la de turbulencia, como son: hostilidad, incertidumbre, declinación, dinamismo, volatilidad, complejidad, crisis, abrupto, munificencia etc., que contribuyen a la falta de unidad conceptual. Algunos de estos términos como hostilidad, crisis, abrupto, declinación munificencia, no complementan la definición de turbulencia, son simplemente tipologías encontradas en la literatura para describir el entorno; otros términos como dinamismo, volatilidad, complejidad, e incertidumbre pueden ser considerados factores que la generan.

Zamutto \& Cameron (1985) y Cameron, Kim \& Whetten (1987) utilizan el concepto declinación para describir una disminución sustancial en los recursos de una organización durante un período de tiempo específico, sin que su entorno necesariamente haya presentado cambios.

Calantone, Schmidt \& Di Benedetto (1997) usan el concepto entorno hostil y lo definen como la cantidad de amenazas que enfrenta una firma debido a la intensidad, vigor y múltiples facetas asumidas por la competencia y por los ciclos de la industria. Algunas características de este entorno son intensidad de precios, producto, distribución y competencia tecnológica, restricciones regulatorias, tendencias demográficas negativas, recortes en personal y materiales. Para Hall (1996), en este entorno se presenta un crecimiento desacelerado en el mercado domestico, presiones inflacionarias sobre costos de distribución y manufactura, presiones a la regula- ción de inversiones y a la conducta de las empresas; la competencia se intensifica, tanto por los competidores actuales como por los nuevos. Covin \& Slevin (1989) han indicado que un entorno hostil se caracteriza por una estructura organizacional con dificultad para predecir los eventos futuros, postura estratégica y desempeño financiero.

Duncan (1972) trabaja el concepto entorno incierto indicando que es aquel en donde existe una incapacidad de la empresa para asignar probabilidades de manera confiable con respecto a la forma como los factores del entorno pueden incidir en el éxito o fracaso de la misma. Sobre el uso del término, Downey \& Slocum (1975) notaron que "incertidumbre" es una palabra tan utilizada comúnmente que es demasiado fácil asumir que se sabe de lo que se está hablando cuando se usa el término. Este término ha sido utilizado tanto como un descriptor del estado de entornos de la organización, así como un descriptor del estado de una persona que se percibe a si misma como carente de información crítica acerca del entorno. Courtney (2001) indica que un mercado con alta incertidumbre presenta estándares tecnológicos cambiantes y competidores que entran y salen en forma constante. Para Terrebery (1968) la incertidumbre es una característica dominante de la turbulencia.

Otro concepto utilizado es el de entorno abrupto (Meyer, 1982; Forbes, Manrakhan \& Banerjee, 2004), entendido como aquel en el cual se presentan perturbaciones transitorias que son difíciles de pronosticar y cuyo impacto en la organización es de generar ruptura y hostilidad. Estas perturbaciones no son amenazas, crisis, catástrofes. No ponen en riesgo la supervivencia de las organizaciones. En este tipo de entorno existe una alta probabilidad de deserción de clientes, baja satisfacción de los consumidores, cambio en regulaciones del gobierno y problemas con organizaciones ecologistas.

Dess \& Beard (1984) mencionan otros tres conceptos adicionales: munificencia; dinamismo, y complejidad. Un entorno munificente (generoso) se define como la escasez o la abundancia de recursos críticos necesitados por una o más firmas que operan dentro de un entorno (Dess \& Beard, 1984, Pfeffer \& Salancik, 1978, Staw \& Szwajkowski, 1975). 
El dinamismo es definido como el cambio en el tiempo de la generosidad y la complejidad (Dess \& Beard, 1984). Se caracteriza por el nivel de cambios presentes en las variaciones constantes de los factores del entorno (Bourgeois, 1985). Para Jhonson \& Scholes (1999) el dinamismo implica que una organización no pueda tomar decisiones futuras basada únicamente sobre datos históricos. Más bien esto debe operar con sus productos actuales mientras se anticipa a los resultados de sus acciones en el futuro. Las organizaciones necesitan considerar la forma como ellos operan en el presente y la forma como la posición en el mercado actual puede afectar lo que pueden hacer en el futuro.

El concepto complejidad se define como la homogeneidad o heterogeneidad de las circunstancias externas que enfrenta una organización (Dess \& Beard, 1984). Representa la medida del número de las configuraciones competitivas que una organización puede considerar como buenas para su estrategia (Chakravarthy, 1997). Para Johnson \& Scholes (1999) la complejidad es el impacto de los elementos del entorno (tecnología sofisticada, diversidad de productos y servicios, incremento en la competencia) sobre la organización.

Esta serie de definiciones ha llevado a algunos autores a indicar que la turbulencia es un fenómeno que se encuentra explicado por una combinación de dimensiones. Gueguen $(1997,2001)$ propuso que la turbulencia (T) está en función de la complejidad (C), dinamismo (D) e incertidumbre (I). $\mathrm{T}=\mathrm{f}(\mathrm{C}, \mathrm{D}, \mathrm{I})$. Este acercamiento se basa en los siguientes argumentos: 1) La turbulencia esta en función de la complejidad y el dinamismo. $\mathrm{T}=\mathrm{f}(\mathrm{C}, \mathrm{D})$. Esta apreciación es compartida por Emery \& Trist (en Dessler, 1992), Terrebery (1968), Ansoff \& Sullivan (1993), Morris, Hansen \& Pitt, (1995) y Chakravarthy, (1997); 2) La incertidumbre se genera por la asociación entre la complejidad y dinamismo (Duncan, 1974; Ganesan, 1994; Daft, Sormusen \& Park, 1988). Para Callot (1997) la complejidad genera incertidumbre. 3) La turbulencia se encuentra vinculada a la incertidumbre y dinamismo. $\mathrm{T}=\mathrm{f}(\mathrm{I}, \mathrm{D})$. Tal como lo expresan Bhattacharya, Jina \& Walson (1996), Ansoff, Eppink \& Gomer (1993). 4) Cameron et al. (1987) argumentan que la turbulencia crea la incertidumbre
( $\mathrm{T}=\mathrm{I})$ y que es el mejor factor de medición de la percepción de la incertidumbre del entorno.

Es importante aclarar que la diferencia entre la primera y segunda combinación presentadas, radica en el uso de los conceptos incertidumbre o turbulencia. Emery \& Trist (1965) a quienes se les atribuye el concepto de turbulencia no buscaron evaluar la capacidad de la empresa de tomar decisiones; sino que su trabajo se orientó a describir el entorno que la rodeaba; razón por la cual no trabajan la incertidumbre. Sin embargo, años más tarde Khandwalla (1972) y Duncan (1974), junto a sus seguidores, se orientan a estudiar el proceso de toma de decisiones para enfrentar entornos con complejidad y dinamismo. Pudiendo decir que esta propuesta, se constituye en un avance para la comprensión del entorno.

Posteriormente Zahrra \& George (2000) y Crossan, Vera \& Nanjad (2001) respaldan el argumento de que la turbulencia se caracteriza por estos tres elementos. Dess \& Beard (1984) y Sull (2009) comparten la idea de que la complejidad y el dinamismo incrementan la turbulencia, pero difieren en el tercer elemento. Para Dess \& Beard (1984) el tercer elemento es la abundancia; para Sull (2009) el tercer elemento es la intensa competencia. La tabla 1 sintetiza estas aproximaciones sobre la relación entre los tres conceptos.

\subsection{Factores que contribuyen a la turbulencia}

En la sección anterior, se hizo una revisión del concepto de turbulencia, llegando a concluir que este fenómeno se genera no sólo por uno, sino por varios eventos que confluyen de manera simultánea $e$ impredecible afectando el desarrollo normal de las actividades del sector generando una respuesta por parte de las organizaciones que lo conforman. Estos elementos son el dinamismo, la incertidumbre y la complejidad. Sin embargo, es importante analizar los factores internos y externas que generan esos tres elementos.

\subsubsection{Factores internos}

Dentro de los factores internos que pueden contribuir a la turbulencia se encuentran las deficiencias 
Tabla 1. Aproximación al concepto de turbulencia y relación con la complejidad, el dinamismo y la incertidumbre².

\begin{tabular}{|c|c|c|}
\hline Autores & propuesta & Expresión \\
\hline $\begin{array}{l}\text { Emery \& Trist, 1965; Terrebery, 1968; Ansoff \& Sullivan, } \\
\text { 1993; Morris, Hansen \& Pitt, 1995; Chakravarthy, 1997) }\end{array}$ & $\begin{array}{l}\text { La turbulencia se encuentra asociada a la comple- } \\
\text { jidad y al dinamismo }\end{array}$ & $T=f(C, D)$ \\
\hline $\begin{array}{l}\text { Khandwalla, 1972; Duncan, 1974; Ganesan, 1994; Daft, } \\
\text { Sormusen \& Park, 1988); Callot, 1997; Johnson \& Scho- } \\
\text { les, } 1999 .\end{array}$ & $\begin{array}{l}\text { La incertidumbre tiene las mismas causas de la } \\
\text { turbulencia. Ambos se deben a la complejidad y } \\
\text { dinamismo del entorno. }\end{array}$ & $I=f(C . D)$ \\
\hline $\begin{array}{l}\text { Bhattacharya, Jina * Walson, 1996; Ansoff, Eppink \& } \\
\text { Gomer, } 1993\end{array}$ & $\begin{array}{l}\text { La incertidumbre asociada al dinamismo genera la } \\
\text { turbulencia del entorno. }\end{array}$ & $T=f(I, D)$ \\
\hline Cameron, Kim \& Whetten, 1987. & $\begin{array}{l}\text { La turbulencia, como resultado de los cambios } \\
\text { rápidos en el medio ambiente conduce a la incer- } \\
\text { tidumbre }\end{array}$ & $I=f(T)$ \\
\hline Sull, 2009. & $\begin{array}{l}\text { La turbulencia esta caracterizada por el dinamis- } \\
\text { mo, la complejidad y la intensa competencia (Co). }\end{array}$ & $T=f(C, D, C o)$ \\
\hline Dess \& Beard, 1984. & $\begin{array}{l}\text { La turbulencia esta caracterizada por el dinamis- } \\
\text { mo, la complejidad y la abundancia }\end{array}$ & $T=f(C, D, A)$ \\
\hline $\begin{array}{l}\text { Gueguen, 1997; Crossan, Zahrra, Ravi \& Goerge, 2000; } \\
\text { Crossan, Vera \& Nanjad, } 2001 .\end{array}$ & $\begin{array}{l}\text { La turbulencia se asocia a la complejidad, el dina- } \\
\text { mismo y la incertidumbre. }\end{array}$ & $T=f(C, D, I)$ \\
\hline
\end{tabular}

en las habilidades del personal, tanto a nivel operativo como directivo (Duncan, 1972; Silverblatt \& Korgaonkar, 1987). Estas deficiencias son en cuanto a formación y experiencia de los empleados; la desarticulación organizacional por falta de coordinación entre metas y objetivos (Emery \& Trist, 1965; Duncan, 1972; Silverblatt \& Korgaonkar, 1987; Venkataraman, Ven, Buckeye, \& Hudson, 1990); cambios permanentes llevados a cabo en la alta dirección de la organización generando incertidumbre en los empleados como lo indican Venkataraman, et al (1990). Adicionalmente se explica la turbulencia en función de las fallas en el proceso de planeación (Grant, 2003) que realizan, evaluando elementos como tipo y duración de planes estratégicos, frecuencia de los planes, gastos de capital, sistema de planeación, etc.

\subsubsection{Factores externos}

Existen factores externos que no dependen de la empresa. Dentro de ellos estan los demográficos y sociales, caracterizados por cambios permanentes en las preferencias y/o necesidades de los clientes dando como resultado un incremento en la densidad de la interacción social provocada por el crecimiento de la población y sus demandas (McCann \& Selsky, 1984; Jaworski \& Kohli, 1993; Droge, Calantone \& Harmancioglu, 2008); los factores estructurales, representados en cambios en el poder de compra de los clientes, variación en los ciclos económicos, incremento de la intensidad de la competencia, rivalidad por precio, saturación en el mercado, existencia de sustitutos, dificultad de predecir ciclos de vida del producto (Duncan, 1972; Dwyer \& Welsh, 1985; Pine II, 1993) lo que dificulta el proceso de obtención de nuevos clientes y mantenimiento de los actuales; turbulencia tecnológica (Dwyer et al 1985; Kuivalainen, Sundqvist, Puumalainen \& Cadogan, 2004; Droge et al 2008); cambios en políticas de regulación gubernamental, lo cual se evidencia en una modificación en la política de restricciones a venta de ciertos productos, regulación a transporte, regulación a precio, protección del medio ambiente, regulación de publicidad, regulación antimonopolio (Dwyer et al 1985; Ulrich \& Wiersema, 1989). Para Caldart \& Ricart (2006) la turbulencia del entorno se debe a cambios en los actores que conforman la

2 Fuente: Elaboración propia resultado de la revisión de literatura. 
industria y cambios en la regulación. Bian (2006), en su tesis doctoral, explora eventos que contribuyen a la turbulencia del entorno identificando tres categorías: sociales, políticos y económicos. La tabla 2 sintetiza lo encontrado en la literatura sobre factores de turbulencia.

Tabla 2. Factores determinantes de la turbulencia ${ }^{3}$.

\begin{tabular}{|c|c|}
\hline Autor & Factores determinantes de la turbulencia \\
\hline Bian (2006) & $\begin{array}{l}\text { Eventos sociales: Exposición mediática (presencia en medios de comunicación), logro de estánda- } \\
\text { res de la industria (Normas de calidad), presencia en redes sociales, donaciones y actividades de } \\
\text { apoyo. } \\
\text { Eventos Políticos: Análisis de relaciones regionales, nacionales e internacionales. } \\
\text { Eventos económicos: Contempla el análisis de política nacional en materia de ciencia y tecnología. }\end{array}$ \\
\hline Craig, T. (1996) & $\begin{array}{l}\text { Cambios demográficos } \\
\text { Cambios sociales } \\
\text { Cambios económicos } \\
\text { Cambios en distribución } \\
\text { Cambios en competencia }\end{array}$ \\
\hline $\begin{array}{l}\text { Droge, C., Calantone, R. \& } \\
\text { Harmancioglu, N. (2008) }\end{array}$ & $\begin{array}{l}\text { Turbulencia de mercado (cambios en preferencias de los clientes, precios, estructura de costos, } \\
\text { composición de competidores) } \\
\text { Intensidad en la competencia } \\
\text { Avances tecnológicos frecuentes }\end{array}$ \\
\hline $\begin{array}{l}\text { Duncan, R. (1972); } \\
\text { Silverblatt, R. \& Korgaonkar, } \\
\text { P. (1987) }\end{array}$ & $\begin{array}{l}\text { Factores Internos: } \\
\text { Recurso Humano (nivel de formación, experiencia tecnológica, habilidades gerenciales, estilos de } \\
\text { comportamiento e interacción con el sistema) } \\
\text { Nivel directivo (características de unidades organizacionales, Relación Staff - Unidades operacio- } \\
\text { nales) } \\
\text { Componente organizacional (objetivos y metas, tipo de producto o servicio } \\
\text { Integración individuo y grupo para obtención de objetivo) } \\
\text { Factores externos } \\
\text { Clientes } \\
\text { Proveedores } \\
\text { Competencia } \\
\text { Componente Socio-político (mecanismos de regulación del gobierno sobre la industria, políticas } \\
\text { públicas, relaciones con sindicatos) } \\
\text { Tecnología (conocimiento de los requerimientos de la industria; mejoramiento y desarrollo de nuevos } \\
\text { productos }\end{array}$ \\
\hline $\begin{array}{l}\text { Dwyer, R. \& Welsh, } \\
\text { A. (1985) }\end{array}$ & $\begin{array}{l}\text { Regulación (estándares del gobierno, restricciones a vendedores, regulación a transporte, regula- } \\
\text { ción a precio, protección del medio ambiente, regulación de publicidad, regulación antimonopolio) } \\
\text { Tecnología(crecimiento de nuevos productos, uso de equipos electrónicos en ventas) } \\
\text { Incertidumbre en la demanda } \\
\text { Cultura (reputación por calidad y servicio) } \\
\text { Competencia (precio, ubicación, calidad, servicio, publicidad) } \\
\text { Disponibilidad de Recursos }\end{array}$ \\
\hline Emery, F. \& Trist, E. (1965) & $\begin{array}{l}\text { Desarticulación organizacional: (grandes organizaciones persiguiendo metas diferentes inmersas en } \\
\text { sociedades con crecimiento constante donde las tasas de crecimiento en investigación y desarrollo } \\
\text { son una constante). } \\
\text { Revolución en comunicación generando sobrecarga en información } \\
\text { Mecanismos de regulación inadecuados para anticiparse a las consecuencias de los cambios }\end{array}$ \\
\hline
\end{tabular}

3 Fuente: Elaboración propia resultado de la revisión de literatura. 


\begin{tabular}{|c|c|}
\hline Autor & Factores determinantes de la turbulencia \\
\hline $\begin{array}{l}\text { Freeman, C. \& Soete, L. } \\
\text { (1997) }\end{array}$ & $\begin{array}{l}\text { Incertidumbre tecnológica } \\
\text { Incertidumbre comercial } \\
\text { Incertidumbre organizacional } \\
\text { Incertidumbre social }\end{array}$ \\
\hline Grant, R. (2003) & $\begin{array}{l}\text { Tipo y duración de planes estratégicos } \\
\text { Frecuencia de los planes } \\
\text { Gastos de capital } \\
\text { Sistema de planeación }\end{array}$ \\
\hline Head, T. (2005) & $\begin{array}{l}\text { Estructura de propiedad } \\
\text { Tipo de industria } \\
\text { Edad de la firma } \\
\text { Número de empleados } \\
\text { Tipo de integración (vertical u horizontal) } \\
\text { Nivel de estandarización, formalización y especialización de la firma } \\
\text { Profesionalización } \\
\text { Cambios económicos } \\
\text { Cambios en el entorno }\end{array}$ \\
\hline $\begin{array}{l}\text { Jaworski, B. \& Kohli, } \\
\text { A. (1993) }\end{array}$ & $\begin{array}{l}\text { Turbulencia de Mercado (cambio en preferencias de clientes, sensibilidad al precio por parte del } \\
\text { cliente, cambios en demanda). } \\
\text { Turbulencia tecnológica (cambios frecuentes en tecnología, difícil pronóstico de nuevas tecnologías; } \\
\text { desarrollos tecnológicos escasos; los nuevos productos se generan con avances tecnológicos). }\end{array}$ \\
\hline $\begin{array}{l}\text { Kuivalainen, O., Sundqvist, } \\
\text { S., Puumalainen, K. \& } \\
\text { Cadogan, J. (2004) }\end{array}$ & $\begin{array}{l}\text { Turbulencia tecnológica } \\
\text { Turbulencia competitiva } \\
\text { Turbulencia del cliente (cambio en preferencias del cliente) } \\
\text { Turbulencia regulatoria (entorno regulatorio) }\end{array}$ \\
\hline $\begin{array}{l}\text { McCann, J. \& Selsky, } \\
\text { J. (1984) }\end{array}$ & $\begin{array}{l}\text { Incremento en la densidad de la interacción social provocada por el Crecimiento de la población y } \\
\text { sus demandas } \\
\text { Crecimiento desigual en la innovación tecnológica. }\end{array}$ \\
\hline Pine II, J. (1993) & $\begin{array}{l}\text { Factores demanda (Capacidad de predecir niveles de demanda, Necesidades cambiantes del clien- } \\
\text { te, cambios en precios, cambios en calidad, cambios en niveles de servicio). } \\
\text { Factores estructurales (Poder de compra del cliente, ciclos económicos, intensidad de la compe- } \\
\text { tencia, rivalidad por precio, saturación en el mercado, existencia de sustitutos, dificultad de predecir } \\
\text { ciclos de vida de producto, alta tasa de cambio tecnológico) }\end{array}$ \\
\hline $\begin{array}{l}\text { Souder, W., Sherman, D. } \\
\text { \& Davies-Cooper, R. (1998) }\end{array}$ & $\begin{array}{l}\text { Ciclo de vida del producto } \\
\text { Desarrollo adecuado de prototipos } \\
\text { Cambios frecuentes en diseño } \\
\text { Efectividad de la inversión en Investigación y desarrollo } \\
\text { Efectividad en lanzamiento de nuevos productos } \\
\text { Certeza en pronóstico de mercado }\end{array}$ \\
\hline $\begin{array}{l}\text { Ulrich, D. \& Wiersema, } \\
\text { M. (1989) }\end{array}$ & $\begin{array}{l}\text { Globalización } \\
\text { Cambios demográficos/sociales } \\
\text { Políticas públicas } \\
\text { Cambios tecnológicos }\end{array}$ \\
\hline $\begin{array}{l}\text { Venkataraman, S., Van de } \\
\text { Ven, A., Buckeye, J. \& } \\
\text { Hudson, R. (1990) }\end{array}$ & $\begin{array}{l}\text { Aspectos financieros (problemas en flujo de cada, adquisición de capital, restructuración de } \\
\text { deuda) } \\
\text { Aspectos de mercadeo (mantener clientes, obtener nuevos clientes, cambio en el foco y estrategia, } \\
\text { buscar nuevos nichos de mercado, decisión de salir del mercado) } \\
\text { Aspectos Organizacionales (cambios en misión y metas; reestructuraciones, reubicaciones) } \\
\text { Aspectos Gerenciales (Cambios en alta dirección, cambios en grupo gerencial) }\end{array}$ \\
\hline
\end{tabular}




\subsection{Efectos de la turbulencia}

Tan importante como analizar los componentes del entorno turbulento, es ver su comportamiento. Ello implica observar su grado de complejidad, dinamismo e incertidumbre. Los tres componentes, inciden en la consecución de resultados por parte de las empresas. Las decisiones asumidas para enfrentar la turbulencia llevarán a las empresas a tener resultados más o menos favorables. Es importante que no se haga una asociación entre turbulencia y resultados negativos. Esta sección presenta efectos positivos y negativos derivados de convivir con esta situación. La sección permite desmitificar la turbulencia; y comprender que puede enfrentarse realizando cambios en las empresas, y lograr resultados favorables.

\subsubsection{Efectos Negativos}

Los primeros trabajos que presentaron los efectos de la turbulencia en las organizaciones se atribuyen a Menninger (1952), Whitney (1962) y Janis (1962), quienes concluyeron que la turbulencia aumenta la incertidumbre de los individuos como resultado de la escasa información disponible, la rigidez en la respuesta y la incapacidad de reacción de la organización. Esta situación tiene impacto en las actitudes de las personas en las organizaciones generando sentimientos de crisis, ansiedad y estrés.

Posteriormente, Zajonc (1965), Khandwalla (1972), Starbuck, Greve \& Hedberg, (1978) y Cameron et al (1987) resaltaron el efecto de la turbulencia sobre el incremento de la centralización en el proceso de toma de decisiones, la búsqueda de la eficiencia a través de la estandarización y la rutinización de los procesos. Cameron et al (1987) indicaron que la organización entra en una predisposición a la planeación a largo plazo, el rechazo a la innovación, la presencia de sentimientos de culpabilidad, el retiro de personal, la baja moral, la generación de conflictos internos y la pérdida de credibilidad de la empresa.

Para Pine III (1993) y Stigter (2003) la turbulencia tiene efectos en fluctuaciones de demanda, ajuste en las características de los productos y servicios ofrecidos y una orientación hacia el desarrollo de nuevos productos que permitan responder a la crisis. Recientemente, algunos autores soportan la idea de que la turbulencia tiene efectos negativos sobre el desempeño (Cameron, Kim \& Whetten, 1987; Tushman \& Anderson, 2001; Lin \& Germain, 2003; Kuivalainen, Sundqvist, Puumalainen \& Cadogan, 2004; Power \& Reid, 2005).

\subsubsection{Efectos Positivos}

La turbulencia no sólo produce riesgos y genera incertidumbre, también es una oportunidad para la generación de rentabilidad y crecimiento de las organizaciones y del sector (Drucker, 1980; Cameron, 1984; Huber, 1984; Tushman \& Anderson, 1986; Hall \& Rosson, 2006; Ayadurai \& Sohail, 2007). Para Stigter (2002) la turbulencia, al generar fluctuaciones en la demanda, obliga a las empresas a adoptar una mayor flexibilidad e innovación para realizar ajustes que permitan desarrollar nuevos productos y servicios. Recientemente Sull (2009) utilizó el concepto "the upside of turbulence" para indicar que la turbulencia crea tres tipos de oportunidades: a) oportunidades para generar productos y servicios con nuevos insumos, b) oportunidad de realizar nuevas combinaciones de recursos y c) oportunidades resultantes de cambios en gustos de las personas.

Para Li \& Atuahene-Gima (2001) o Kuivalainen et al. (2004), las medidas de turbulencia pueden estar basadas en las percepciones de los ejecutivos más que en las características del entorno. Lo anterior se puede identificar en estudios que presentan diferencias entre las medidas objetivas y subjetivas para evaluar el cambio en el entorno (Boyd, Dess \& Rasheed, 1993; Tosi, Aldag \& Storey, 1973). Castrogiovanni (1991) y Boyd et al. (1993) manifestaron que existen modelos de comportamiento gerencial o algunas acciones que pueden utilizarse como medidas del entorno pero que los modelos de desempeño organizacional pueden ser medidas objetivas. La tabla 3 nos enseña las consecuencias de la turbulencia por diferentes autores. 
Tabla 3. Consecuencias de la turbulencia ${ }^{4}$

\begin{tabular}{|c|c|}
\hline Autor & Efectos de la turbulencia \\
\hline $\begin{array}{l}\text { Menninger, 1952; Whitney, 1962; } \\
\text { Janis, } 1962\end{array}$ & $\begin{array}{l}\text { La turbulencia tiene un impacto negativo en la empresa, ya que incrementa la incertidumbre } \\
\text { de los individuos, y genera sentimientos de crisis, ansiedad y estrés. }\end{array}$ \\
\hline $\begin{array}{l}\text { Zajonc, 1965; Khandwalla, 1972; } \\
\text { Starbuck, Greve \& Hedberg, } \\
\text { 1978; Cameron et al, } 1987\end{array}$ & $\begin{array}{l}\text { Manifiestan que la turbulencia lleva a la empresa a un incremento en la centralización, bus- } \\
\text { cando una orientación a la búsqueda de la eficiencia, la estandarización y la rutinización; } \\
\text { pero el proceso de toma de decisiones se lleva a cabo en pocas personas, sin tener en } \\
\text { cuenta la opinión de personas de otros niveles jerárquicos. }\end{array}$ \\
\hline $\begin{array}{l}\text { Cameron, K., Kim, M. \& } \\
\text { Whetten, D. (1987) }\end{array}$ & $\begin{array}{l}\text { Se da una orientación a la centralización en toma de decisiones, no planeación a largo } \\
\text { plazo, resistencia a la innovación, sentimientos de culpabilidad, renuncias y despidos, resis- } \\
\text { tencia al cambio, baja moral, pérdida de credibilidad, aumento de conflictos internos. }\end{array}$ \\
\hline Pine, 2003; Stigter, 2003 & $\begin{array}{l}\text { La turbulencia genera fluctuaciones permanentes en la demanda, generando incertidumbre } \\
\text { en los mercados: Se presentan ajustes en productos y servicios; cambios en desarrollo de } \\
\text { nuevos productos. }\end{array}$ \\
\hline $\begin{array}{l}\text { Cameron, Kim \& Whetten, 1987: } \\
\text { Tushman y Anderson, 2001; } \\
\text { Li \& Atuahene-Gima, 2001; } \\
\text { Lin \& Germain, 2003; } \\
\text { Kuivalainen, Sundqvist, } \\
\text { Puumalainen \& Cadogan, 2004; } \\
\text { Power \& Reid, 2005. }\end{array}$ & $\begin{array}{l}\text { La turbulencia puede llevar a efectos negativos en el desempeño de las organizaciones; al } \\
\text { incrementarse el dinamismo en el sector, que derive en guerra de precios, imitación de los } \\
\text { productos y servicios; e implementación de soluciones de corto plazo. }\end{array}$ \\
\hline Boyne \& Meir, 2009 & $\begin{array}{l}\text { Efecto negativo en el desempeño de las organizaciones como consecuencia de los cam- } \\
\text { bios internos, y que para controlar la volatilidad debe mantenerse la estabilidad estructural. }\end{array}$ \\
\hline
\end{tabular}

\subsection{Valoración de la turbulencia}

Para poder determinar si un sector está atravesando por una situación de turbulencia, y no por momentos de hostilidad, declinación, crisis, es necesario hacer una valoración del entorno. Para ello se deben estudiar las características de incertidumbre, dinamismo y complejidad. En esta sección se hace una revisión de los principales mecanismos utilizados para valorar la turbulencia del entorno. La tabla 4 incluye una variedad de posibilidades; que se convierten en alternativas que pueden ser utilizadas por las personas encargadas de monitorear el entorno de las empresas para determinar si existe o no turbulencia.

4 Fuente: Elaboración propia resultado de la revisión de literatura. 
Tabla 4. Valoración de la turbulencia del entorno 5

\begin{tabular}{|c|c|c|c|c|}
\hline autor & año & $\begin{array}{l}\text { revis- } \\
\text { ta }\end{array}$ & $\begin{array}{l}\text { Dimensiones: } \\
\text { I: Incertidumbre } \\
\text { D: Dinamismo } \\
\text { C: Complejidad } \\
\text { T: Turbulencia }\end{array}$ & Medidas \\
\hline $\begin{array}{l}\text { Akgun, Byrne, } \\
\text { Lynn \& Keskin }\end{array}$ & 2007 & JET-M & $\mathrm{D}$ & $\begin{array}{l}\text { Usan a Jaworski \& Kohli (1993). Evalúan turbulencia de mercado me- } \\
\text { diante dos preguntas, y turbulencia tecnológica mediante } 4 \text { preguntas. }\end{array}$ \\
\hline $\begin{array}{l}\text { Becherer \& } \\
\text { Maurer }\end{array}$ & 1998 & ETP & D & $\begin{array}{l}\text { Utilizan Covin, Slevin (1989) y Miller, Friesen (1983) para medir el dina- } \\
\text { mismo del entorno. }\end{array}$ \\
\hline $\begin{array}{l}\text { Beesley \& } \\
\text { Hamilton }\end{array}$ & 1984 & JIE & $\mathrm{D}$ & $\begin{array}{l}\text { Utilizan la siguiente ecuación para medir turbulencia } T=[(I B+ \\
\text { ID) +(DB+DD)] / Stock. Siendo (IB) independent births; dependent bir- } \\
\text { ths (DB); independent deaths (ID) y dependent deaths (DD) }\end{array}$ \\
\hline Bian & 2006 & IESE & C & $\begin{array}{l}\text { Utiliza un estudio de caso longitudinal, pero se vale de una combina- } \\
\text { ción entre un caso base y diagramas causales para interpretar la forma } \\
\text { como las empresa logran competir con éxito. }\end{array}$ \\
\hline Bourgeois & 1985 & AMJ & I & $\begin{array}{l}\text { Utilizan Duncan (1972), con modificaciones en la manera de medir cada } \\
\text { componente del entorno. }\end{array}$ \\
\hline & & & $\mathrm{D}$ & Miden la variación en el crecimiento de las ventas en el mismo período. \\
\hline Boyd & 1990 & SMJ & C & $\begin{array}{l}\text { Utiliza Aldrich (1979), para medir la homogeneidad y la concentración } \\
\text { mediante el Herfindahl. Suma de cuadrados de cuotas de mercado de } \\
\text { todas las empresas en un sector. Competencia perfecta si es } 0 \text {, si } 1 \\
\text { infinita cuota de mercado. }\end{array}$ \\
\hline $\begin{array}{l}\text { Brown \& } \\
\text { Khirchoff }\end{array}$ & 1997 & FER & $\mathrm{D}$ & $\begin{array}{l}\text { Se usan } 4 \text { ítems de Miller \& Friesen (1983), para dinamismo y } 3 \text { de } \\
\text { Khandwalla (1976-1977) para evaluar hostilidad. }\end{array}$ \\
\hline Caldart, \& Ricart & 2003 & IESE & $D ; C$ & $\begin{array}{l}\text { Realizan una modelación de las variables dinamismo y complejidad. } \\
\text { Del nivel de análisis es la estrategia a nivel corporativo. Para modelar di- } \\
\text { namismo siguen a Mintzberg (1983), quien considera este componente } \\
\text { como la medición de los factores impredecibles de una empresa; y para } \\
\text { la variable complejidad siguen a Siggelkow \& Rivkin (2005). }\end{array}$ \\
\hline $\begin{array}{l}\text { Cameron, Kim } \\
\& \text { Whetten }\end{array}$ & 1987 & AMJ & $\mathrm{T}$ & $\begin{array}{l}\text { Utilizan cinco medidas para evaluar la turbulencia. 1) La suma del por- } \\
\text { centaje de cambio para cada año, 2) La desviación estándar, 3) el co- } \\
\text { eficiente de variación, 4) el coeficiente de fluctuación, 5) el coeficiente } \\
\text { de alienación medido durante } 6 \text { años. }\end{array}$ \\
\hline Covin \& Slevin & 1989 & SMJ & $\mathrm{T}$ & $\begin{array}{l}\text { Basados en Khandwalla (1976-1977) para medir hostilidad (similar a tur- } \\
\text { bulencia) teniendo en cuenta las variables de seguridad, oportunidades } \\
\text { de inversión, oportunidad de mercadeo y control. }\end{array}$ \\
\hline Craig & 1996 & OS & $\mathrm{D}$ & $\begin{array}{l}\text { Mide tendencias demográficas, cambios sociales, cambios económi- } \\
\text { cos, cambios en distribución, cambios en competencia. }\end{array}$ \\
\hline
\end{tabular}

5 Fuente: Elaboración propia resultado de la revisión de literatura. Convenciones de las revistas: AMJ: Academy of Management Journal, ASQ: Administrative Science Quarterly, CJofAS: Canadian Journal of Administrative Science, ETP: Entrepreneurship, Theory and Practice, FER: Frontiers of Entrepreneurship Research, HBSP: Harvard Business School Press, IndMarkM: Industrial marketing Management, IESE: IESE Business School, JBEth: Journal of Business Ethics, JBR: Journal of Business Research, JED: Journal of Developmental Entrepreneurship, JET-M: Journal of Engineering and Technology Management, JIE: Journal of Industrial Economics, JMS: Journal of Management Studies, JofAMS: Journal of the Academy of Marketing Science, JofBV: Journal of Business Venturing, JofM: Journal of Marketing, JofMR: Journal of Marketing Research, JofPIM: Journal of Product Innovation Management, JSBM: Journal of Small Business Management, MS: Management Science, OAS: Organizations and Administrative Sciences, OS: Organizations Science, PhD: PhD Dissertation, SMJ: Strategic Management Journal. 


\begin{tabular}{|c|c|c|c|c|}
\hline $\begin{array}{l}\text { Daft, Sormusen } \\
\& \text { Parks }\end{array}$ & 1988 & SMS & I, C & $\begin{array}{l}\text { Se mide incertidumbre estratégica percibida }(I E P)=I(C+T C) \text {, donde I } \\
\text { es la importancia percibida en el sector; } C \text { es la complejidad percibida } \\
\text { del sector y Tc, es la tasa de cambio del sector. Para medir incertidum- } \\
\text { bre tienen en cuenta } 6 \text { factores: tecnología, economía, competidores, } \\
\text { clientes, regulación, sociales-culturales. }\end{array}$ \\
\hline $\begin{array}{l}\text { Davis, Morris } \\
\text { \& Allen }\end{array}$ & 1991 & $\begin{array}{l}\text { JofA- } \\
\text { MS }\end{array}$ & $\mathrm{D}$ & $\begin{array}{l}\text { Mide el cambio tecnológico, la competencia entre firmas y la tasa de } \\
\text { crecimiento de la industria. }\end{array}$ \\
\hline \multirow{2}{*}{$\begin{array}{l}\text { Dess, Lumpkin } \\
\& \text { Covin }\end{array}$} & \multirow{2}{*}{1997} & \multirow{2}{*}{ SMJ } & D & $\begin{array}{l}\text { Del instrumento de Miller (1983), usa } 3 \text { ítems para medir el nivel de di- } \\
\text { namismo del entorno. }\end{array}$ \\
\hline & & & I & $\begin{array}{l}\text { Hace una combinación de dinamismo e imprevisibilidad. Esta última la } \\
\text { mide con } 2 \text { variables de Miller (1983). }\end{array}$ \\
\hline \multirow{3}{*}{ Dess \& Beard } & \multirow{3}{*}{1984} & \multirow{3}{*}{ ASQ } & $C, D$ & Mira el grado en el que el cambio en una industria afecta a otra. \\
\hline & & & $\mathrm{D}$ & $\begin{array}{l}\text { Compuesta de estabilidad e inestabilidad. Mediante } 5 \text { variables miden } \\
\text { esta dimensión. }\end{array}$ \\
\hline & & & C & Compuesta de homogeneidad y heterogeneidad. \\
\hline $\begin{array}{l}\text { Droge, Calantone } \\
\text { \& Harmancioglu }\end{array}$ & 2008 & JofPIM & $\mathrm{D}$ & $\begin{array}{l}\text { Mide la turbulencia de mercado, tecnológica y de rivalidad. Utiliza el } \\
\text { instrumento de Miller \& Droge (1986), Khandwalla (1976-1977) y Miller } \\
\text { (1983). }\end{array}$ \\
\hline \multirow[b]{2}{*}{$\begin{array}{l}\text { Downey, Hellriegel } \\
\text { \& Slocum }\end{array}$} & \multirow[b]{2}{*}{1975} & \multirow[b]{2}{*}{ ASQ } & I & $\begin{array}{l}\text { Utiliza el instrumento de Lawrence \& Lorsch (1967), para estudiar } 9 \\
\text { ítems en tres categorías: la falta de claridad en la información, la in- } \\
\text { certidumbre generada por la relación y la alta amplitud existente entre } \\
\text { acción y resultado. }\end{array}$ \\
\hline & & & I & $\begin{array}{l}\text { Utiliza de Duncan (1972), para medir tres características: falta de infor- } \\
\text { mación sobre los factores asociados con la toma de decisiones am- } \\
\text { bientales, la falta de conocimiento sobre las consecuencias de una de- } \\
\text { cisión en la organización, la capacidad de juzgar el efecto de un factor } \\
\text { sobre la organización. }\end{array}$ \\
\hline \multirow{5}{*}{ Duncan } & \multirow{5}{*}{1972} & \multirow{5}{*}{ ASQ } & I & Falta de información de los factores del entorno (6 ítems). \\
\hline & & & I & $\begin{array}{l}\text { La falta de conocimiento sobre las consecuencias de una decisión so- } \\
\text { bre la organización. }\end{array}$ \\
\hline & & & I & $\begin{array}{l}\text { La capacidad de juzgar el efecto de un factor sobre la organización con } \\
\text { cierto nivel de certeza. }\end{array}$ \\
\hline & & & C & $\begin{array}{l}\text { Dos alternativas simple o compleja. Se mira el número de factores que } \\
\text { intervienen en una decisión y el número de componentes. }\end{array}$ \\
\hline & & & $\mathrm{D}$ & $\begin{array}{l}\text { Dos sub-dimensiones: el grado en que los factores que son estables } \\
\text { y la frecuencia con la que decisiones teniendo en cuenta los nuevos } \\
\text { factores. }\end{array}$ \\
\hline Dwyer \& Welsh & 1985 & JofMR & I & $\begin{array}{l}\text { Un instrumento que contiene } 6 \text { características del entorno (presentadas } \\
\text { por Acrol, Reve \& Stern,1983) Los actores desarrollados son regulación } \\
\text { y cultura. Los demás factores tecnología, demanda, competencia y re- } \\
\text { cursos ya estaban presentados en otros cuestionarios. }\end{array}$ \\
\hline Glazer \& Weiss & 1993 & JofMR & I,D & Usa simulación para evaluar cambios e incertidumbre. \\
\hline Grant & 1996 & SMJ & $\mathrm{D}, \mathrm{I}$ & $\begin{array}{l}\text { Se indaga mediante entrevistas a estos funcionarios sobre proceso de } \\
\text { planeación, estructura y rol de la estrategia corporativa, el rol del pro- } \\
\text { ceso de planeación, link planeación estratégica y otro sistema de toma } \\
\text { de decisión. }\end{array}$ \\
\hline Hall \& Rosson & 2006 & JBEth & । & Mide la incertidumbre tecnológica, comercial, organizacional y social, \\
\hline $\begin{array}{l}\text { Ireland, Hitt, } \\
\text { Bettis \& Porras }\end{array}$ & 1987 & SMJ & I & Usan a Miles \& Snow (1978). \\
\hline
\end{tabular}




\begin{tabular}{|c|c|c|c|c|}
\hline Jaworski \& Kohli & 1993 & JofM & $\mathrm{D}$ & $\begin{array}{l}\text { Miden tres aspectos: turbulencia de mercado (Tm), intensidad de la } \\
\text { competencia (Ic), y turbulencia tecnológica (Tt). Usan } 17 \text { ítems. Para } \\
\text { Tm evalúan cambios en gustos de clientes; Con (Ic) evalúa el compor- } \\
\text { tamiento, recursos y habilidades de los rivales, (Tt) estudia el cambio en } \\
\text { la tecnología de la industria. }\end{array}$ \\
\hline Khandwalla & 1974 & ASQ & $\mathrm{D}$ & $\begin{array}{l}\text { Utiliza un instrumento para medir el cambio en la tecnología utilizada } \\
\text { por la firma. }\end{array}$ \\
\hline Khandwalla | & $\begin{array}{l}1976- \\
1977\end{array}$ & OAS & $\mathrm{D} ; \mathrm{C}$ & $\begin{array}{l}\text { Mide la turbulencia mediante el dinamismo y la complejidad (heteroge- } \\
\text { neidad). Concibe el dinamismo como cambios tecnológicos, culturales } \\
\text { y económicos; la dificultad en predecir cambios del entorno; la expan- } \\
\text { sión de los mercados; y el cambio cíclico. La heterogeneidad se mide } \\
\text { teniendo en cuenta la diversidad de los mercados, cambios en gustos } \\
\text { de clientes. }\end{array}$ \\
\hline $\begin{array}{l}\text { Kuivalainen, } \\
\text { Sundqvist, } \\
\text { Puumalainen } \\
\& \text { Cadogan }\end{array}$ & 2004 & $\begin{array}{l}\text { CJO- } \\
\text { fAS }\end{array}$ & D & $\begin{array}{l}\text { Se basan en Jaworski \& Kohli (1993), para medir turbulencia de mer- } \\
\text { cado (Tm), intensidad de la competencia (Ic) y turbulencia tecnológica } \\
\text { (Tt) pero incluye la dimensión de regulación basados en Dwyer \& Welsh } \\
\text { (1985). }\end{array}$ \\
\hline Lawless \& Finch & 1989 & SMJ & $\mathrm{D}, \mathrm{C}$ & Usan a Dess \& Beard (1984) \\
\hline $\begin{array}{l}\text { Lawrence } \\
\text { \& Lorsch }\end{array}$ & 1967 & & I & $\begin{array}{l}\text { Utiliza } 3 \text { ítems de } 7 \text { puntos para mirar la incertidumbre a través de: [ne- } \\
\text { cesidad de los departamentos de producción, investigación, marketing } \\
+ \text { grado de dificultad para llevar a cabo las acciones de cada depar- } \\
\text { tamento + tiempo de respuesta para determinar el éxito del uso de los } \\
\text { recurso de los departamentos]. }\end{array}$ \\
\hline Lichtenthaler & 2009 & AOM & D & $\begin{array}{l}\text { Se basa en Jaworski \& Kholi (1993), para medir dinamismo (cambio } \\
\text { tecnológico y cambio de mercado). }\end{array}$ \\
\hline Lindsay \& Rue & 1980 & AMJ & C & $\begin{array}{l}\text { Se basa en Duncan (1972). Mide la complejidad interna y externa, } \\
\text { siendo } C=(\Sigma I) \times\left(\Sigma F_{3}\right)^{2} \text {. Donde (I) es el numero ítems seleccionados } \\
\text { (máximo 25); (F) es el numero de diferentes categorías en las que se ha } \\
\text { marcado frecuentemente al menos una vez. }\end{array}$ \\
\hline Matthews \& Scott & 1995 & JSBM & I & $\begin{array}{l}\text { Evaluar el estado de incertidumbre: la imposibilidad de predecir el esta- } \\
\text { do del entorno a través la falta de información. }\end{array}$ \\
\hline Miles \& Snow & 1978 & McGraw & & $\begin{array}{l}\text { Evalúa la incertidumbre con dos dimensiones homogeneidad-heteroge- } \\
\text { neidad y estabilidad-cambio. }\end{array}$ \\
\hline Miller & 1983 & MS & $\mathrm{D}, \mathrm{C}$ & Mide el dinamismo, la hostilidad y la heterogeneidad de la firma. \\
\hline Miller & 1992 & OSC & I & Utiliza a Khandwalla (1976-1987) redefinido por Miller (1988). \\
\hline \multirow[t]{2}{*}{ Miller } & \multirow[t]{2}{*}{1988} & \multirow[t]{2}{*}{ AMJ } & $\mathrm{D}$ & $\begin{array}{l}\text { La imprevisibilidad y dinamismo están muy correlacionados con la in- } \\
\text { certidumbre (Miller \& Droge, 1986). }\end{array}$ \\
\hline & & & I & Dinamismo x Imprevisibilidad. \\
\hline Miller \& Droge & 1986 & ASQ & $\mathrm{I}, \mathrm{D}$ & $\begin{array}{l}\text { Utiliza los instrumentos de Khandwalla (1974) y Miller (1983) para medir } \\
\text { el grado de cambio e incertidumbre en los mercados y tecnología. }\end{array}$ \\
\hline Miller \& Friesen & 1983 & SMS & D & $\begin{array}{l}\text { Mide el estado previsible y estable de las actividades de los compe- } \\
\text { tidores y las preferencias de los consumidores, y la innovación en el } \\
\text { mercado. }\end{array}$ \\
\hline Milliken & 1987 & AMJ & I & $\begin{array}{l}\text { Clasifica la incertidumbre en tres tipos: estado, estado, efecto y res- } \\
\text { puesta. Se basa en Duncan (1972). }\end{array}$ \\
\hline Naman \& Slevin & 1993 & SMJ & T & $\begin{array}{l}\text { Utiliza } 8 \text { items: } 5 \text { de Miller \& Friesen (1982); } 3 \text { de Khandwalla (1977). La } \\
\text { escala de } 7 \text { puntos. }\end{array}$ \\
\hline Pine II & 1993 & HBSP & I & $\begin{array}{l}\text { Diseña un instrumento llamado mapa de turbulencia para evaluar la tur- } \\
\text { bulencia de mercado en términos de la inestabilidad, incertidumbre y } \\
\text { falta de control de las firmas en el mercado. Utiliza información demo- } \\
\text { gráfica, percepción de entorno de mercado; información de las empre- } \\
\text { sas y procesos y percepciones organizacionales. }\end{array}$ \\
\hline
\end{tabular}




\begin{tabular}{|c|c|c|c|c|}
\hline Sawyerr & 1993 & SMJ & I & $\begin{array}{l}\text { Usa a Daft, Sormusen \& Park (1988). Tres variables para medir Incerti- } \\
\text { dumbre percibida: Tasa de cambio del entorno, nivel de complejidad, } \\
\text { grado de dependencia del sector. }\end{array}$ \\
\hline $\begin{array}{l}\text { Silverblatt, \& } \\
\text { Korgaonkar }\end{array}$ & 1987 & JBR & $\mathrm{C}$ & $\begin{array}{l}\text { Utiliza instrumento de Lindsay \& Rue (1980) modificado. El instrumento } \\
\text { se divide en } 6 \text { factores del entorno ( } 16 \text { preguntas) y } 3 \text { internos ( } 12 \text { pre- } \\
\text { guntas). Una escala que mide para cada factor la frecuencia de ocu- } \\
\text { rrencia y la importancia. } \\
C=(I) \times(F) 2 \text {. Donde (I) corresponde al número de ítems seleccionados } \\
\text { de los } 28 \text { disponibles y (F) es el número de factores diferentes marca- } \\
\text { dos al menos con una calificación de } 3 \text { (frecuentemente). }\end{array}$ \\
\hline Slater \& Narver & 1994 & JofM & $\mathrm{D}$ & $\begin{array}{l}\text { Usa Miller (1987), para evaluar dinamismo y magnitud de los cambios } \\
\text { (turbulencia de mercado y tecnología). }\end{array}$ \\
\hline Souder \& Song & 1997 & JofPIM & $\mathrm{D}$ & $\begin{array}{l}\text { Mide la incertidumbre de mercado (conocimiento de las necesidades } \\
\text { del cliente, trasladas cambio en necesidades a diseño de nuevos pro- } \\
\text { ductos). }\end{array}$ \\
\hline Sutcliffe \& Huber & 1998 & SMJ & $\mathrm{C}$ & Adaptación de Dess \& Beard (1984). \\
\hline $\begin{array}{l}\text { Tushman \& } \\
\text { Anderson }\end{array}$ & 1986 & ASQ & I & $\begin{array}{l}\text { Se mira el pronóstico de crecimiento de la demanda - el crecimiento } \\
\text { de la demanda actual | x } 100 \text { / Crecimiento de la demanda actual. El } \\
\text { proceso se hace para } 5 \text { años. }\end{array}$ \\
\hline $\begin{array}{l}\text { Venkataraman, } \\
\text { Van de Ven, } \\
\text { Buckeye\&Hudson }\end{array}$ & 1990 & JofBV & $\mathrm{D}$ & $\begin{array}{l}\text { Mide el dinamismo a través de la valoración del desempeño como cre- } \\
\text { cimiento de ventas, empleados, ranking de desempeño en la industria. }\end{array}$ \\
\hline
\end{tabular}

\subsubsection{Valoración de la complejidad del entorno}

La complejidad descansa sobre la idea que los factores del entorno que afectan las decisiones de una organización son numerosos y heterogéneos (Thompson, 1967; Terreberrry, 1968; Child, 1972). Las metodologías para medir la complejidad van desde técnicas cualitativas como el estudio de caso, hasta análisis cualitativo. En este caso, dentro de los instrumentos más utilizados para medir complejidad en investigaciones de turbulencia se encuentran los de Khandwalla (1976/1977), Dess \& Beard (1984) y Sutcliffe \& Huber (1998). En todos ellos, las preguntas están orientadas a comprender el grado de diversidad o de homogeneidad de las empresas en categorías como: comprensión del entorno, vínculos con otras empresas, forma de percibir los cambios del entorno, tipo de clientes y mercados, líneas de productos y servicios ofrecidos, nivel de sofisticación tecnológica requerida en el sector.

Estos instrumentos se basan principalmente en Duncan (1974) quien para medir complejidad identifica diversos factores y componentes de entorno (interno y externo). El entorno externo, tiene cinco componentes: clientes, proveedores, competidores, socio-político, tecnológico. ¿Y los del entorno interno? Mide la complejidad contando el número y heterogeneidad de los factores tomados en consideración en una decisión, con la siguiente fórmula: $\mathrm{F}$ x $\mathrm{C}^{2}$ (siendo $\mathrm{F}$ y $\mathrm{C}$ factores y componentes del entorno). Un caso particular, es el de Aldrich (1979), quien utiliza el Herfindahl Index para medir el nivel de concentración de la industria.

\subsubsection{Valoración de la Incertidumbre de entorno}

La incertidumbre corresponde a la falta de información sobre los factores del entorno, lo que hace imposible predecir el impacto de una decisión sobre la organización (Morris, Hansen \& Pitt, 1995). Para Duncan (1972) la incertidumbre se determina por la suma de tres dimensiones: falta de información entre el medio ambiente y la toma de decisiones, falta de conocimiento sobre el resultado de una decisión, la capacidad para dar una probabilidad de ocurrencia de eventos. Milliken (1990) otorga nombre a estos tres tipos de incertidumbre: incertidumbre de estado, incertidumbre de efecto e incertidumbre de respuesta. 
Para medir la incertidumbre se han utilizado principalmente los instrumentos diseñados por Lawrence \& Lorch (1967) y Duncan (1972). Otro grupo de trabajos, usan las propuestas de Miles \& Snow (1978) y Miller (1983). Ambas posibilidades evalúan la disponibilidad y calidad en la información que tienen las empresas para pronosticar cambios del entorno (regulaciones, tecnología, demografía) que puedan afectar su desempeño.

\subsubsection{Valoración del dinamismo del entorno}

Un entorno dinámico es concebido por Khandwalla (1976/1977) como aquel en el que se presentan cambios permanentes en tecnología, cultura y economía que afectan el comportamiento de las empresas. Al evaluar el dinamismo se examinan los cambios en el entorno pero no se pretende estudiar la naturaleza de estos cambios (Gueguen, 2001). Aun cuando existen numerosos instrumentos para medir dinamismo, estos se basan principalmente en los trabajos de Khandwalla (1976/1977), Miller \& Friesen (1982) y Jaworski \& Kohli (1993), quienes exploran la intensidad de la competencia, la tecnología, los cambios en el mercado y los cambios en procedimientos.

Se utilizan instrumentos con preguntas orientadas a identificar el nivel de cambio en factores tecnológicos, culturales, políticos, demográficos, regulatorios, etc; que afectan la decisión de las empresas de implementar nuevos productos y servicios. Igualmente se hace un levantamiento de información sobre variaciones de productos y servicios; niveles de precios, inversiones en publicidad llevados a cabo por los competidores del sector. Los estudios realizados presumen que a mayor numero de productos, mayor dinamismo. La valoración del dinamismo, se hace principalmente mediante estudios longitudinales, que permitan ver la evolución del sector en un lapso de tiempo no menor a cinco años. El método de casos, es una de las alternativas preferidas para estudiar el dinamismo.

\subsection{Alternativas utilizadas por las empresas para enfrentar la turbulencia}

Para Makridakis (1979), enfrentar la turbulencia es prácticamente imposible, teniendo que cuenta la dificultad en pronosticar las discontinuidades que se presentan en el entorno. Sin embargo, para algunos autores sí es posible hacerlo. Respecto a esta última línea se encuentran en la literatura cuatro enfoques. El primero surge en la década de los sesenta, en las publicaciones realizadas por Burns \& Stalker (1961), Lawrence \& Lorsch (1967), Thompson (1967), Terreberry (1968), Child (1972 y 1975), Duncan (1972), Khandwalla (1972 y 1974) y Jurkovich (1974). Estos autores recomiendan estudiar la interacción entre el ambiente y la organización para realizar cambios orientados a lograr un balance entre estructura organizacional, liderazgo, recurso humano y cultura corporativa. Se hace necesario crear una estructura organizacional flexible y descentralizada, que pudiera balancear y generar equilibrio entre una organización mecánica y una sistémica. Otros autores, recomiendan promover el comportamiento ético basado en la tolerancia y buscar la interrelación con otras organizaciones (Emery \& Trist, 1965; Tereberry, 1968; Metcalfe, 1974; Metcalfe \& Mc Quillan, 1977; Post \& Mahon, 1980; Mc Cann \& Selsky, 1984; Morris et al, 1995). Emery \& Trist (1965:76) indican que una estrategia de adaptación para los entornos turbulentos es la creación de matrices organizacionales, que son organizaciones interconectadas que pueden maximizar la cooperación, donde ninguna organización puede realizar el rol de las demás.

El segundo enfoque recomienda hacer frente al entorno turbulento llevando a cabo actividades de emprendimiento que promuevan la creatividad y la generación de ideas innovadoras para el desarrollo de nuevos productos que rompan con la rutina y lo tradicional (Ansoff, 1965, 1990; Davis et al, 1991; Miller et Friesen, 1982; Naman, \& Slevin, 1993; D'Aveni, 1995; Morris et al, 1995; Mason, 2006).

Un tercer enfoque, esta conformado por autores que recomiendan enfrentar la turbulencia mediante la modificación al proceso de planeación estratégica realizado por la empresa. Se debe dar una orientación orientada al desarrollo de cambios incrementales, enfocados en la experimentación más que en la formulación. La definición de las estrategias debe surgir de un proceso espontáneo logrado gracias a una auto-organización y procesos de aprendizaje permanente; lo que le permita a la empresa ser 
más sensitiva y orientada a la acción para generar sorpresas estratégicas y construir la resiliencia, con lo que se generen rupturas o transformaciones (Camillus \& Datta, 1991; Stacey, 1995; Brown \& Eisenhardt, 1997; Volberda, 1997; Pascale, 1999)

Un cuarto enfoque para enfrentar la turbulencia tiene que ver con el desarrollo de capacidades dinámicas que permitan a la organización hacer frente a los factores que generan la turbulencia. Concretamente se recomienda desarrollar habilidades de comunicación, enfocarse en resultados, alinear los valores de la organización, ser capaz de tomar decisiones sin información perfecta, desarrollar competencias emocionales, lograr la focalización y flexibilidad y construir reservas y concentración de recursos (Chakravarthy, 1997; Narasimha, 2001; Souba, 2003; Bitar, 2003; Tan, Li \& Li, 2006; Suikki, Tromstedt \& Haapasalo, 2006; El Sawy, 2008).
Dentro de este mismo enfoque, se encuentra la propuesta de D'Aveni (1994), quien indica que la turbulencia obliga a las empresas a reaccionar de manera rápida y agresiva para crear nuevas ventajas si no quieren perder su posición en el mercado. Para Prokresch (1993), el potencial de introducción de innovaciones o cambios del medio ambiente es un factor clave de éxito porque la empresa debe producir inestabilidad. Una posición ecléctica es la presentada por Rigby $(2001)^{6}$, quien recomienda que primero debe identificarse la fuente de la turbulencia (micro económica, macro económica, tecnológica, competitiva, regulatoria), luego recomienda (1) actuar rápidamente frente a las condiciones de mercado sin reaccionar de forma exagerada; (2) focalizarse en el producto o servicio central, (3) definir planes de acción alternativos en las épocas de crecimiento y (4) en las épocas de recesión económica confiar en los planes de contingencia y no cambiar la estrategia. La tabla 5 sintetiza lo encontrado en la literatura sobre cómo enfrentar la turbulencia.

Tabla 5. Propuestas teóricas para enfrentar la turbulencia ${ }^{7}$

\begin{tabular}{|c|c|}
\hline Autores & Propuesta \\
\hline $\begin{array}{l}\text { Emery, F. \& Trist, E. (1965); Metcalfe, J. (1974); } \\
\text { Metcalfe, L. \& McQuillan, W. (1977); McCann, J. \& } \\
\text { Selsky, J. (1984), Post \& Mahon (1980), }\end{array}$ & Gestión intra-organizacional, regulación externa. \\
\hline Terreberry, S. (1968) & Interrelación entre las organizaciones. \\
\hline Morris et al (1996) & Comportamiento ético, fortalecer valores. \\
\hline Bonis (1972) & Control del entorno. \\
\hline $\begin{array}{l}\text { Naman et Slevin (1993), Morris et al. (1995), } \\
\text { Davis et al. (1983), Miller \& Friesen (1983), } \\
\text { Ansoff, I. (1968 y 1990); Mason (2006) }\end{array}$ & $\begin{array}{l}\text { Estrategia de emprendimiento (innovación e introducción del cambio, reacción } \\
\text { rápida y flexible). }\end{array}$ \\
\hline D’Aveni, R. (1994), Prokresch (1993) & Perturbar la acción. \\
\hline & Comportamiento creativo. \\
\hline $\begin{array}{l}\text { Brisson (1992), Mintzberg (1990), Jennings } \\
\text { \& Seaman (1994) }\end{array}$ & Estructura orgánica. \\
\hline Polley, D. (1997) & Desarrollo de la tolerancia y de una estructura flexible. \\
\hline Kalika (1985) & Descentralización. \\
\hline
\end{tabular}

6 Realizó un análisis de 377 compañías que entraron en recesión en Estados Unidos entre 1990-1991, de las cuales el 31\% para el año 2001 presentaban incremento en el valor de sus acciones.

7 Fuente elaborada por el autor. 


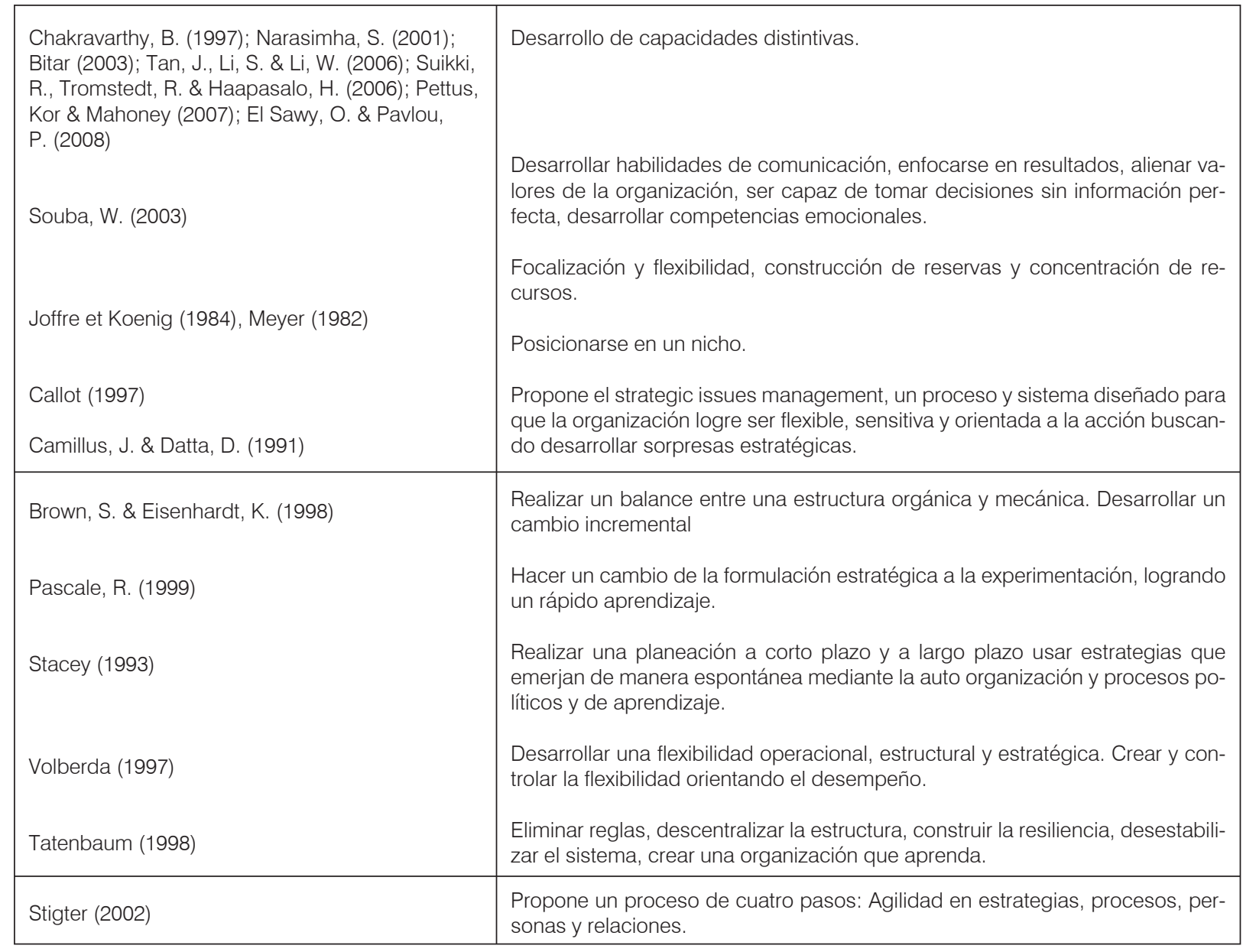

A pesar de las numerosas reflexiones teóricas y trabajos empíricos sobre la turbulencia del entorno, en los que se encuentran definiciones, características, formas de medición, causas, consecuencias y recomendaciones para enfrentar el fenómeno, no existe una única definición del concepto, falta uniformidad en las dimensiones que caracterizan la turbulencia y no se ha encontrado evidencia específica que permita sistematizar las pautas comunes de comportamiento de las organizaciones ante la presencia de turbulencia. Sin embargo, en estos trabajos se encontraron elementos que permitieron describir el fenómeno y que se representan en la Ilustración 1.

Se puede observar cómo al hablar de turbulencia del entorno nos referimos a un conjunto de acon- tecimientos transformadores constantes e impredecibles provenientes de factores demográficos, tecnológicos y de regulación. Como consecuencia de estos cambios, el entorno se vuelve inestable (dinámico), la capacidad de previsión por parte de las empresas cada vez tiene menos certeza (incertidumbre) y la comprensión del entorno es cada vez más difícil dada la heterogeneidad de actividades que allí se presentan (complejidad); esta situación afecta el normal desempeño del sector (clientes, competidores, proveedores, distribuidores). Surgen cambios en preferencias de los clientes, rivalidad por promociones y precios, cambio en composición de competidores, saturación en el mercado y aparición de sustitutos. 


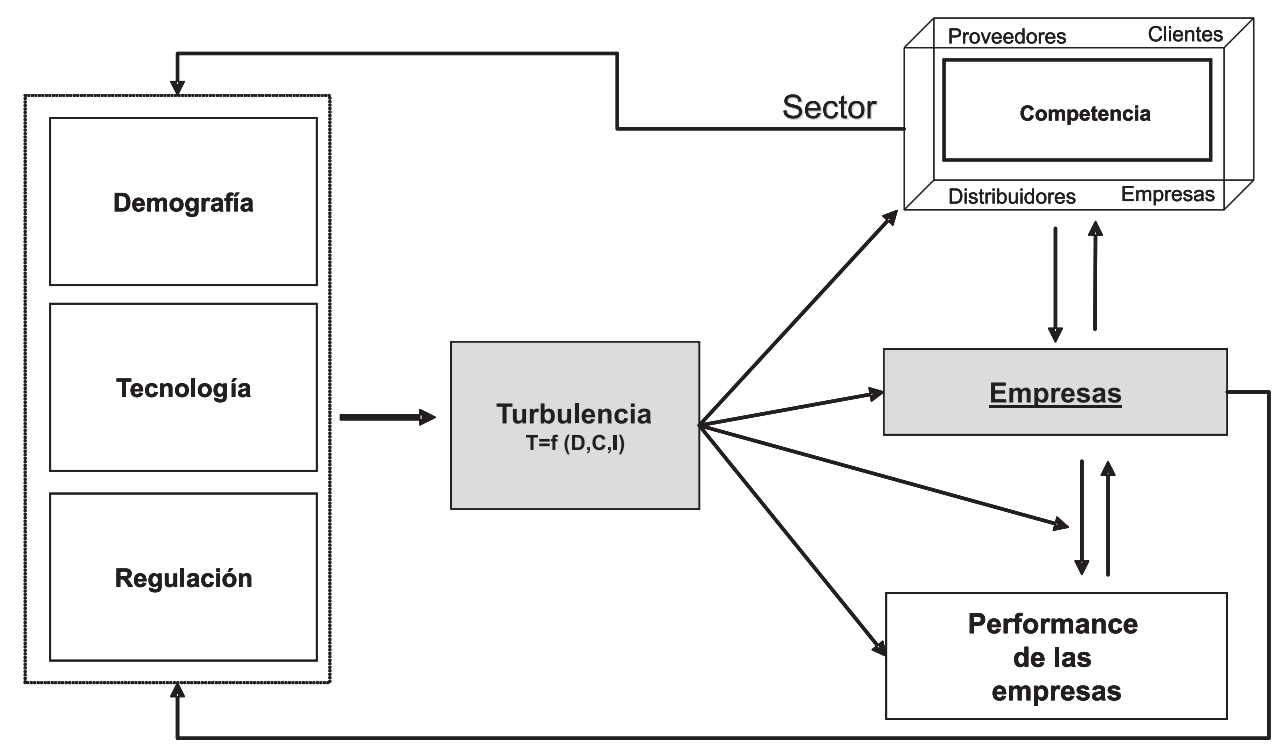

Figura 1. El fenómeno de la turbulencia ${ }^{8}$

Ante estos cambios, una de las alternativas que tienen las empresas para enfrentar la turbulencia es la de modificar sus estrategias buscando ventajas competitivas temporales. Estos cambios a nivel corporativo, directivo y organizacional pueden realizarse en varios campos: fortalecimiento de la relación organización-entorno, promover el emprendimiento y la innovación, generar capacidades dinámicas, y desarrollar una flexibilidad estratégica. Estos ajustes pueden generar efectos contraproducentes como son el estrés, la ansiedad, la incertidumbre, la resistencia al cambio o la pérdida de credibilidad. Sin embargo, dado que los cambios buscan la eficiencia, la turbulencia se convierte en un reto y oportunidad de crecimiento.

Al hacer la contrastación con las demás alternativas presentadas en el documento, puede indicarse que el cambio estratégico es una alternativa integradora; e incorpora elementos de las demás propuestas para enfrentar la turbulencia. Al implementarlo, se hace necesario llevar a cabo una interrelación entre las organizaciones, efectuar un control permanente del entorno; desarrollar un comportamiento creativo que lleve a que se generen distorsiones; es necesario aplicar una flexibilidad operacional, aprender rápidamente, etc. Recientemente, Ricart (2010) indica que para tomar el mando del sector y enfrentar los elementos propulsores del cambio, es necesario implementar el cambio estratégico para generar un nuevo modelo de negocios.

El desempeño de las empresas tiene un efecto feedback sobre las estrategias, las cuales a su vez pueden afectar el comportamiento del sector. En cuanto a los factores generadores de discontinuidades (regulación, tecnología, demografía) pueden verse afectado por el cambio presentado en las empresas y en el sector. La situación descrita indica que un entorno vive en permanente turbulencia. Se refleja una co-evolución dinámica entre firmas y entorno (Lewin \& Volverda, 1999), donde las empresas persiguen sin tregua ventajas competitivas transitorias.

Para enfrentar la turbulencia y aprovechar las oportunidades tecnológicas, demográficas, de regulación

8 Elaboración propia. 
$\mathrm{u}$ otras, se hace necesario tomar medidas integrales, no basta con implementar soluciones para el dinamismo, o la complejidad, o la incertidumbre. Por ello, se toma partido por el cambio estratégico como mecanismo para enfrentar la turbulencia. La perspectiva seleccionada permite hacer una revisión de los efectos que tienen las decisiones de política, de activos y de gobierno para lograr generar un nuevo modelo de negocios. Y adicionalmente permite evaluar el impacto hacia el sector y los factores externos generadores de turbulencia.

Aunque se ha realizado una breve descripción del cambio estratégico como alternativa para enfrentar la turbulencia del entorno, se hace necesario explorar con mas detenimiento el concepto de cambio estratégico, sus orígenes, los avances en sus investigaciones, y lo mas importante para el presente documento, elementos que permitan a las empresas utilizarlo como mecanismo de intervención para enfrentar la turbulencia del entorno.

\section{El concepto de cambio estratégico}

El estado del entorno, es una variable que incide sobre los resultados financieros de las empresas, mas específicamente sobre la rentabilidad, el flujo de efectivo o el EVA. Este impacto tiene una intensidad que varia dependiendo de la empresa analizada y la estrategia que ésta adopte. Por otro lado, la dirección del impacto de las diferentes variables y actores puede ser positiva o negativa. Ante esta situación, las empresas tienen que responder adecuadamente para lograr perpetuarse en un ambiente que cambia de manera permanente.

Explicar por qué las organizaciones cambian ha sido un tema abordado ampliamente por la disciplina de la administración ${ }^{9}$ (Van de Ven \& Poole, 1995), en especial en discusiones sobre comportamiento organizacional, teoría organizacional y estrategia. Andrews (1980) indicó que una empresa está cambiando siempre en respuesta su propio carácter y desarrollo pasado.
Aun cuando el tema no es novedoso, si se hace necesario estudiarlo teniendo en cuenta los cambios en la estrategia que implementan las empresas buscando hacer frente a un entorno cada vez más complejo, dinámico e incierto. Para Mintzberg, Ahlstrand \& Lampel (1998) el interés por estudiar el cambio radica en la preocupación tanto por identificar los factores que lo originan como por la necesidad de proponer alternativas que permitan enfrentarlo de una mejor manera.

La teoría organizacional y el comportamiento organizacional se han preocupado por estudiar el cambio estructural de las organizaciones y los cambios a nivel individual o grupal. Por su parte, la estrategia de acuerdo con Kraatz \& Zajac (2001) muestra el modo a través del cual las organizaciones logran adaptarse a los cambios competitivos, tecnológicos y sociales que ponen en peligro su supervivencia.

Algunas definiciones de estrategia guardan relación con la idea de cambio. Para Ansoff (1965) la estrategia es la forma que tiene la empresa de transformar una posición actual en una posición futura descrita por los objetivos, sujeta a las restricciones de capacidad y potencia. Hofer \& Schendel (1978) denominan estrategia a las características básicas del encaje que una organización logra con su entorno. Para Argyris (1985) o Steiner \& Miner (1985) la estrategia es una respuesta a las fuerzas y debilidades del ambiente interno y a las oportunidades y amenazas presentes en el entorno. Porter (1991) indica que la estrategia es el acto de alinear la empresa y su entorno. Esta idea es compartida por Johnson \& Scholes (1999) quienes manifiestan que la estrategia es el medio de adecuación de las actividades de una organización con el entorno en el que opera. Más recientemente Ramos-Rodríguez \& Ruiz-Navarro (2004) y Nag, Hambrick \& Chen 2007), buscando unificar conceptos, indican que el entorno es uno de los principales temas trabajados en la estrategia y la razón es que este puede afectar la supervivencia de la empresa.

9 Las principales publicaciones que abordan el cambio organizacional y estratégico son: Journal of Change Management, Journal of Organizacional Change, Strategic Change, Journal of Accounting and Organizacional Change, Researh in Organizational Change, Business and Environment, Business Strategy and Environment,y Strategic Management Journal. 
Teniendo en cuenta la importancia que tiene el cambio estratégico para las organizaciones, se presentan a continuación diferentes definiciones sobre el concepto buscando tener claridad sobre los elementos que conforman dicho concepto. Es importante anotar que la literatura sobre cambio estratégico no se encuentra apoyada en un modelo analítico unificado, por el contrario, existen diferentes teorías. Uno de los pioneros en el estudio del cambio fue Lewin (1951) quien desarrolló una teoría de cambio social en la que se definen a las instituciones sociales como el resultado de un conjunto dinámico de fuerzas opuestas (positivas o negativas) que dirigen o restringen el cambio. Para Lewin el cambio se define como un proceso de aprendizaje a través del cual el sistema llega al nuevo equilibrio temporal que se busca. Años más tarde, Bennis (1966) estudió el cambio desde dos puntos de vista: el primero denominado "theories of change" que se enfoca en identificar la forma como las organizaciones cambian y los factores generadores del cambio; el segundo denominado "theories of changing" identifica la forma como el cambio puede ser gerenciado en las organizaciones. Esta sería una diferencia con lo planteado por Lewin (1951) al considerar que el cambio podría ser administrado.

En los años posteriores, la reflexión sobre cambio estratégico se concentró en los factores generadores del cambio y en la realización de ajustes a las definiciones ya existentes, con un elemento que parece ser compartido por muchos autores como es alineación con el entorno. Child (1972) hizo énfasis en el papel de los gerentes de las empresas en el proceso de monitoreo de los cambios en el entorno y posterior modificación de los lineamientos estratégicos de la empresa para lograr la coherencia interna y externa.

Para Hofer \& Schendell (1978), Tichy (1982) y Chaffee (1985) la supervivencia de una empresa depende de la alineación existente entre los componentes organizacionales (misión y estrategia, procesos, personal, etc.) con las áreas críticas de tecnología, política interna (ejercicio del poder) y cultura. Un elemento adicional en el tema del cambio estratégico lo proporcionan Dutton \& Duncan (1987) quienes dividen el proceso en dos etapas: formulación (etapa en la que se identifica la necesidad de introducir una modificación) y posteriormente la implementación o institucionalización del cambio.

La década de los noventa trae nuevos elementos al estudio del cambio surgiendo propuestas concretas para enfrentarlo; Gioia, Thomas, Clark \& Chittipeddi (1994) establecen que deben redefinirse la misión y propósitos de la organización o hacer un cambio sustancial en metas y prioridades. A mediados de la década, Van de Ven \& Poole (1995) presentan una de las definiciones mas utilizadas en la literatura sobre cambio estratégico, para ellos cambio estratégico es una diferencia en la forma, calidad o estado a lo largo del tiempo de la alineación de una organización con su entorno externo. Los autores parten de la idea desarrollada por Hofer \& Schendel (1978), que el cambio permite alcanzar los objetivos de la empresa, al constituirse en un patrón de asignación de recursos presentes y planificados de la empresa, al igual que de las interacciones que ésta realiza con el entorno. Un par de años después, Rajagopalan \& Spreitzer (1997), van mas allá de la definición de Van de Ven \& Poole (1995), al proponer una teoría de cambio estratégico en la que estudian el cambio estratégico desde tres perspectivas: racional, aprendizaje y cognitivo. La racional estudia los cambios en el contenido de la estrategia; aprendizaje explora adicionalmente cambios en las condiciones del entorno; y la cognitiva estudia las estructuras de conocimiento de los directivos usadas para tomar decisiones. Como resultado de esta integración, proponen que el cambio estratégico depende del aprendizaje y adaptación de los mapas directivos, consecuencias del proceso, de los cambios organizativos y de los efectos de las acciones directivas.

Otras de las dimensiones trabajadas en el cambio estratégico son la magnitud y la frecuencia, las cuales se constituyen en temas transversales, ya que ha sido abordadas desde la década de los años setenta. Watzlawick, Weakland \& Fish (1974), introdujeron la clasificación de cambio de primer y segundo orden. En el de primer orden se presentan modificaciones pequeñas en algunos aspectos de la estrategia; los cambios de segundo orden son revolucionarios y modifican el contenido de la estrategia. Posterior- 
mente Quinn (1980), indicó que el cambio se va forjando a través del tiempo, y las iniciativas de cambio estratégico surgen en la gerencia de forma incremental, a partir de decisiones internas y eventos externos que afectan subsistemas estratégicos. Nadler \& Tushman (1989), hablaron de cambios incrementales, anticipatorios, estratégicos y reactivos. Luego Brown \& Eisenhardt (1997), entendieron el cambio como un proceso en el que largos períodos de movimientos incrementales son interrumpidos por cortos períodos de ajuste radicales. Años después Weick \& Quinn (1999), diferenciaron el cambio entre episódico y continuo. El primero es un cambio discontinuo, poco esporádico e intencional, mientras el cambio continuo es acumulativo y evolutivo.

Los nuevos elementos incluidos en el estudio del cambio permitieron explorar otras facetas del concepto. En los primeros años del siglo XXI surgen definiciones que integran los factores generadores del cambio, así como la manera de enfrentarlo. Sosa (2003) define cambio estratégico como la variación a lo largo del tiempo de la alineación de la organización con su entorno que surge y responde a las interpretaciones directivas de los acontecimientos externos y/o internos de la organización y a la búsqueda de la opción más adecuada para tal realineación, y que se traduce en el cambio en la misión o la alteración del su enfoque para lograr la visión, propiciando una modificación en el contenido de la estrategia de la empresa, y en ocasiones, un cambio en los sistemas, las estructuras y/o cultura de la misma empresa.

Otros autores como Fiss \& Zajac (2006) presentan un concepto alternativo del cambio estratégico que involucra un proceso de decisión iniciado a nivel interno. Esta posición destaca el papel de los tomadores de decisiones, quienes ante las discontinuidades del entorno ó ante problemas internos de la compañía, consideran opciones de cambio en su posición en el sector y su relación con proveedores, clientes y competidores e introducen una nueva visión de la compañía. Para estos autores el cambio estratégico es visto no solo como un cambio es estructura y procesos, sino como una reorientación cognitiva de la organización. Tal cambio indica el repasar la puesta al día y la ejecución de una estrategia existente tal como la extensión de programas existentes, centros de estudio y ajustes para vencer presiones ambientales, competencia y la amenaza de identidad.

Dufour \& Steane (2006) clasifican el cambio estratégico en cuatro perspectivas teóricas: clásica, contingente, política y comportamental. La perspectiva clásica se basa en la idea que el cambio es una actividad técnica, no política que surge como respuesta a directrices propuestas por los líderes. La perspectiva contingente percibe el cambio como un proceso de ajuste entre las propuestas de la organización y el entorno y la forma de realizar el cambio es a través del diseño de una estructura organizacional que se adapte al entorno. La perspectiva comportamental se basa en la idea que el cambio se origina en variables de tipo individual. Finalmente, en la perspectiva política el cambio estratégico implica un proceso planeado en términos de estructura, sistemas e influencia de comportamiento.

Ricart (2010) menciona tres tipos de cambios estratégicos que se presentan para generar nuevos modelos de negocios: Los verticalmente superiores, los de nicho, y los de competencia horizontal. El primero de ellos, opera como lo haría una tecnología más avanzada; hacer lo mismo, pero de una manera más efectiva. El cambio conocido como nicho, busca apostar a un segmento inexplorado o desatendido; y finalmente, la alternativa de competencia horizontal implica competir sin realizar cambios, llevando a la empresa a imponerse o convivir como tercera opción durante cierto tiempo. La tabla 6 que reúne diferentes aproximaciones alrededor del concepto de cambio estratégico y organizacional propuestas en los últimos 60 años por estudios de la administración.

\subsection{El cambio estratégico y la turbulencia del entorno}

En la sección anterior se indicó que un mecanismo para enfrentar la turbulencia del entorno es la implementación del cambio estratégico por parte de las empresas. Se hará una argumentación que permita identificar cuál es el beneficio de esta alternativa, y las fases requeridas para llevar a cabo el cambio estratégico. 
Tabla 6. Aproximaciones al concepto de cambio ${ }^{10}$

\begin{tabular}{|c|c|c|c|}
\hline Autor & Año & Fuente & DEFINICIONES Y COMPONENTES DE CAMBIO \\
\hline Lewin & 1951 & LBR & $\begin{array}{l}\text { El cambio es un proceso progresivo, que se cumple en tres fases denominadas descongelar, } \\
\text { modificar y congelar. }\end{array}$ \\
\hline Ansoff & 1965 & LBR & $\begin{array}{l}\text { Cambio estratégico es cuando una empresa reajusta su posición de productos y mercados } \\
\text { así como la distribución de sus recursos en ellos. }\end{array}$ \\
\hline Bennis & 1966 & LBR & $\begin{array}{l}\text { Presenta una diferencia entre "theories of change" and "theories of changing". La primera se } \\
\text { preocupa por los factores generadores de cambio,y la manera de cambiar por parte de las } \\
\text { organizaciones; la segunda estudia la gerencia del cambio. }\end{array}$ \\
\hline $\begin{array}{l}\text { Hofer \& } \\
\text { Schendel }\end{array}$ & 1978 & LBR & $\begin{array}{l}\text { Se entiende el cambio estratégico como la modificación en el contenido de la estrategia de } \\
\text { una empresa, teniendo en cuenta el ámbito de aplicación, el despliegue de recursos, venta- } \\
\text { jas competitivas y la sinergia. }\end{array}$ \\
\hline Quinn & 1980 & $\mathrm{MQ}$ & $\begin{array}{l}\text { El cambio se va forjando a través del tiempo, y el cambio se da de manera incremental a } \\
\text { partir de decisiones internas y eventos que afectan subsistemas estratégicos. }\end{array}$ \\
\hline Tichy & 1982 & OD & $\begin{array}{l}\text { Cambio estratégico es la realienación de los aspectos técnicos, políticos y culturales que } \\
\text { implican cambios en los sistemas de productos, servicios, mercados, estructura organizativa } \\
\text { y recursos humanos. }\end{array}$ \\
\hline Hambrick, & 1983 & AMJ & El cambio estratégico es una reforma de la organización en miembros, activos y procesos. \\
\hline Gray y Ariss & 1985 & AMR & $\begin{array}{l}\text { Es el cambio en la misión global de una empresa o unidad independiente; o la alteración } \\
\text { radical de su enfoque para conseguir esta misión. }\end{array}$ \\
\hline Chaffee & 1985 & AMR & $\begin{array}{l}\text { El cambio estratégico es cómo las empresas perciben su posición en términos de ajuste y } \\
\text { cómo la empresa altera internamente su posición para conseguir un ajuste más cercano al } \\
\text { entorno que le rodea. }\end{array}$ \\
\hline $\begin{array}{l}\text { Reimann } \\
\& \text { Wiener }\end{array}$ & 1986 & $\mathrm{AOM}$ & $\begin{array}{l}\text { El cambio estratégico se concibe como la adaptación a condiciones cambiantes del entor- } \\
\text { no. }\end{array}$ \\
\hline Lorch & 1986 & CMR & $\begin{array}{l}\text { El cambio estratégico es el repensamiento básico de las creencias con las que la empresa } \\
\text { define y lleva a cabo sus negocios }\end{array}$ \\
\hline Boeker & 1989 & AMJ & $\begin{array}{l}\text { El cambio estratégico es la forma como las organizaciones se ajustan a los cambios del } \\
\text { entorno }\end{array}$ \\
\hline $\begin{array}{l}\text { Nadler \& } \\
\text { Tushman }\end{array}$ & 1989 & AME & $\begin{array}{l}\text { Proponen cuatro tipos de cambio estratégico: Incremental, estratégico, anticipatorio, reac- } \\
\text { tivo. }\end{array}$ \\
\hline $\begin{array}{l}\text { Gioia, \& } \\
\text { Chittipeddi, }\end{array}$ & 1991 & SMJ & El cambio estratégico es una redefinición de la misión y propósitos de la organización. \\
\hline $\begin{array}{l}\text { Van de Ven } \\
\text { \& Poole }\end{array}$ & 1995 & AMR & $\begin{array}{l}\text { Cambio organizacional es la diferencia en la forma, calidad o estado a lo largo del tiempo de } \\
\text { la alineación de una organización con su entorno. }\end{array}$ \\
\hline $\begin{array}{l}\text { Barnett \& } \\
\text { Carroll }\end{array}$ & 1995 & ARS & $\begin{array}{l}\text { Cambio estratégico es la transformación de una organización entre dos momentos en el } \\
\text { tiempo. Involucra elementos de estructura de la empresa. }\end{array}$ \\
\hline $\begin{array}{l}\text { Rajagopalan } \\
\text { \& Spreitzer }\end{array}$ & 1997 & AMR & $\begin{array}{l}\text { El cambio estratégico es una modificación en la alienación de la organización con su entorno, } \\
\text { abarcando cambios en el contenido de la estrategia, y cambios en el entorno externo que } \\
\text { propician iniciar e implementar cambios en el contenido de la estrategia. }\end{array}$ \\
\hline Dunphy & 1996 & $\mathrm{HR}$ & $\begin{array}{l}\text { Cambio estratégico es la manera como la organización planea su modificación como resul- } \\
\text { tado de la incapacidad de adaptarse a las discontinuidades del entorno. }\end{array}$ \\
\hline
\end{tabular}

10 Fuentes revisadas: AME: Academy of Management Executive, AMR: Academy of Management Review, AOM: Academy of Management Journal, ARS: Annual Review of Sociology, AROS: American Review of Psychology, ASM: Advances in Strategic Management, CMR: California Management Review, HR: Human Relations, JBEM: Journal of Business Economic and Management, JBS: Journal of Business Studies, JOCM:: Journal of Organizational Change Management, LBR: Libro, MQ: The McKinsey Quarterly, OCL: organizational Change and Learning, OD: Organizational Dynamics, OS: Organization Science, PAR: Public Administration Review, PhD: PhD Dissertation, SBR: Schmalenbach Business Review, SMJ: Strategic management journal. 


\begin{tabular}{|c|c|c|c|}
\hline $\begin{array}{l}\text { Mullins \& } \\
\text { Cummins }\end{array}$ & 1999 & JOCM & $\begin{array}{l}\text { Cambio estratégico es el ajuste en el contenido de la estrategia de la empresa, en términos } \\
\text { de los mercados a los que se dirige, las tecnologías que emplea y/o los clientes a los que } \\
\text { sirve, suponiendo al mismo tiempo cambios en los sistemas, las estructuras y/o cultura de } \\
\text { la empresa. }\end{array}$ \\
\hline Weick \& Quinn & 1999 & AROS & $\begin{array}{l}\text { Cambio estratégico son los ajustes en curso que realiza una empresa y que aunque pueden } \\
\text { ser pequeños, son frecuentar y continuos y capaces de alterar su estructura y estrategia. }\end{array}$ \\
\hline Markoczy & 2000 & JBS & $\begin{array}{l}\text { Cambio estratégico es la reorientación el los procesos de la empresa y en algunos casos } \\
\text { modifica su core. }\end{array}$ \\
\hline $\begin{array}{l}\text { Gordon, } \\
\text { Stewart, Sweo } \\
\& \text { Luker }\end{array}$ & 2000 & JOM & $\begin{array}{l}\text { Cambio estratégico es la adaptación de la organización a las condiciones cambiantes del } \\
\text { entorno. }\end{array}$ \\
\hline Kraatz \& Zajac & 2001 & OS & $\begin{array}{l}\text { Cambio estratégico es la alteración de la posición estratégica para conseguir un mejor ajus- } \\
\text { te al entorno competitivo cambiante, que con frecuencia amenaza la supervivencia de una } \\
\text { compañía. }\end{array}$ \\
\hline Leker & 2001 & SBR & $\begin{array}{l}\text { Indica que el cambio estratégico es la reorientación de la empresa en un entorno competitivo } \\
\text { que ha estado acompañado de deterioro del desempeño. }\end{array}$ \\
\hline Sosa & 2003 & PhD & $\begin{array}{l}\text { Cambio estratégico es definido como la variación a lo largo del tiempo de la alineación de la } \\
\text { organización con su entorno, que surge y responde a las interpretaciones directivas de los } \\
\text { acontecimientos externos y/o internos de la organización y a la búsqueda de la opción más } \\
\text { adecuada para tal realineación. }\end{array}$ \\
\hline $\begin{array}{l}\text { Langley \& } \\
\text { Denis }\end{array}$ & 2006 & OCL & $\begin{array}{l}\text { El cambio estratégico se concibe como los cambios que involucran el core técnico, medio } \\
\text { ambiental y operacional de la compañía. }\end{array}$ \\
\hline Fiss \& Zajac & 2006 & AMJ, & $\begin{array}{l}\text { Cambio estratégico es la modificación en la alineación de la organización con los factores del } \\
\text { entorno. Para ellos, el cambio estratégico es visto no solo un cambio es estructura y proce- } \\
\text { sos, sino como una reorientación cognitiva de la organización. }\end{array}$ \\
\hline Radovic & 2008 & JBEM & $\begin{array}{l}\text { Cambio estratégico se define como la implementación de nuevos procedimientos o tecno- } \\
\text { logías con los cuales se busca alinear a la compañía con las demandas de su entorno o } \\
\text { capitalizar en sus oportunidades de negocio. }\end{array}$ \\
\hline
\end{tabular}

Es importante partir de lo mencionado recientemente por Kotler \& Caslione (2010) quienes postulan que la turbulencia, es ahora la condición normal de las industria, mercados y compañías. Para ellos, este fenómeno es la nueva normalidad, y la función de quienes se encuentran al frente de las organizaciones es implementar mecanismos para enfrentarla, utilizando en algunos casos una armadura defensiva, y en otros una decisión orientada al aprovechamiento de las oportunidades.

La responsabilidad que tiene quien se encuentre al frente de una organización para enfrentar la turbulencia es importante. De acuerdo con Tamayo, Gutiérrez \& Martínez (2007), los entornos dinámi$\cos$ y repletos de discontinuidades contribuyen a la constante necesidad de cambio por parte de los dirigentes de las organizaciones, ellos al ser los encargados de percibir la necesidad de un cambio es- tratégico, planificarlo y ponerlo en marcha. De esta manera el cambio puede ser un generador de ventaja competitiva, ya que las organizaciones pueden anticiparse a los cambios del entorno y responder a esos cambios de un modo efectivo y eficiente. Para Andrews (1980) una de las funciones del directivo es la de adaptar la empresa a los cambios de su entorno.

Ricart (2010), indica que los propulsores del cambio no tendrán capacidad de influir en los sectores al menos que los directores de empresa decidan modificar su propuesta de valor, aprovechando alguna de estas discontinuidades para repensar su modelo de negocio. En definitiva, no es la tecnología sino su adopción; no es la posibilidad del cambio, sino el cambio mismo cuando éste se transforma en un modelo de negocio que se adapte diferente a las nuevas reglas o, mejor aún, crear nuevas reglas. $\mathrm{Si}$ 
esto se realiza, de acuerdo con Kotler y Caslione (2010) se evitaría seguir la sugerencia mencionada por Greenspan (2007) que para enfrentar la turbulencia, hay que arreglársela como se pudiera y rezar.

\subsubsection{Cómo llevar a cabo el cambio?}

Al observar los cambios que se presentan en un sector, tendemos a identificar las fuerzas que los generan. No hay duda que la tecnología influye, los cambios en gustos de las personas, así como los cambios regulatorios o las fuerzas de la globalización, y todos ellos configuran fronteras distintas a los mercados.

Para Sosa (2003) aunque el cambio es generalmente impuesto por el entorno, algunas empresas lo ge- neran a su interior y lo utilizan como una fuente de refuerzo, que le permita dominar su actividad y su futuro y para mejorar las condiciones a sus empleados, entendiéndose la decisión de cambiar como el resultado de un proceso de reflexión iniciado internamente.

De acuerdo con Rajagopalan \& Spreitzer (1997) las condiciones del entorno llevan a los gerentes de las empresas a efectuar cambios en el contenido de la estrategia para alcanzar resultados organizacionales. El cambio se concibe como un proceso iterativo, donde el aprendizaje se constituye en un elemento que permitirá a la empresa tener un mejor entendimiento del entorno y su impacto en la organización. La Ilustración 2 presenta la forma como a través del cambio estratégico se puede enfrentar la turbulencia.

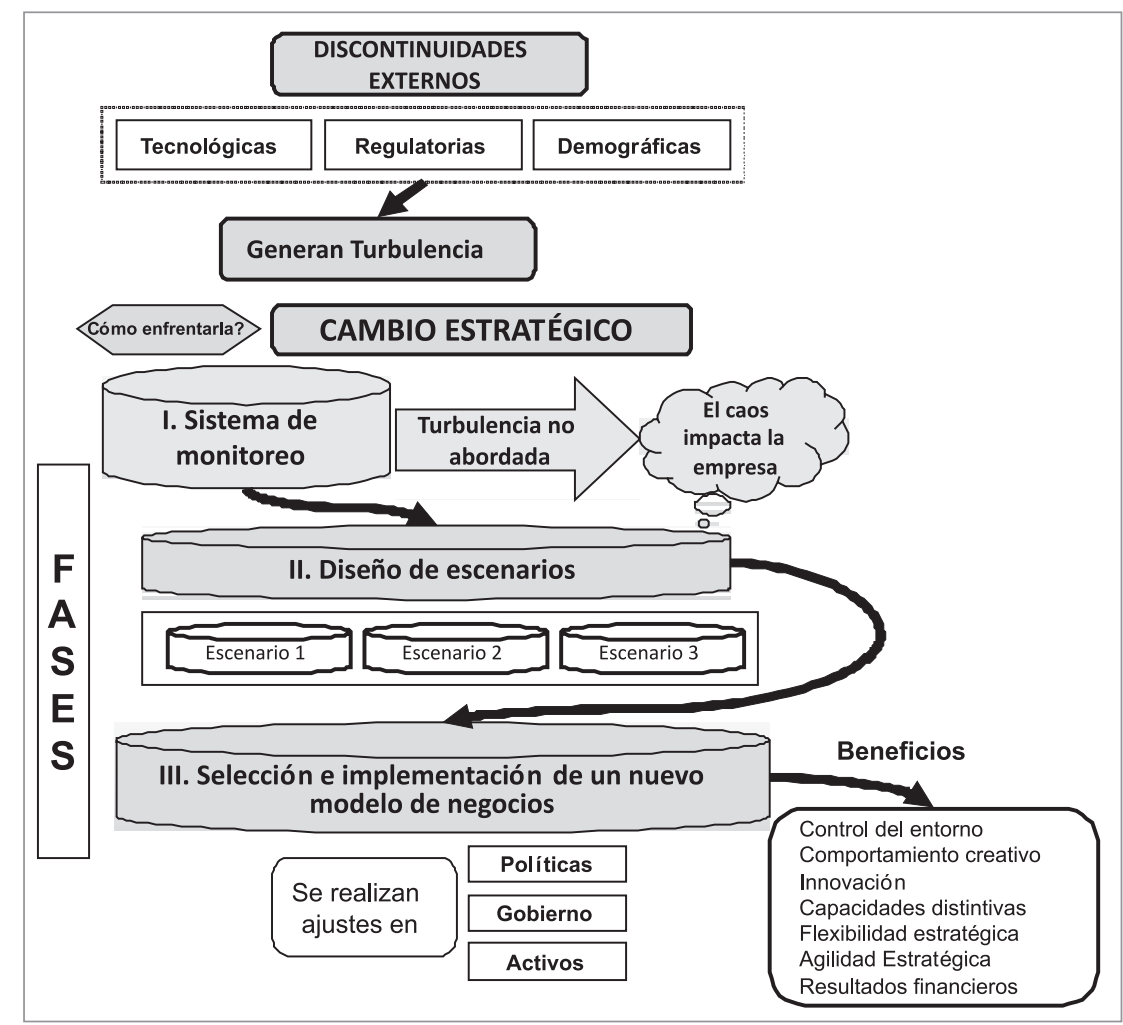

Ilustración 2. Enfrentando la turbulencia con cambio estratégico ${ }^{11}$

11 Elaboración propia. 
Las discontinuidades generadas por fuera de la empresa contribuyen a la turbulencia, y para dar solución a esta situación, los directivos de las empresas deben estar conscientes de la necesidad de llevar a cabo un cambio en su modelo de negocios. De acuerdo con Anderson \& Paine (1975), sin esta consciencia, un estimulo del entorno podría ser considerado irrelevante para la organización, y tener un impacto negativo en los resultados de la compañía.

Una vez se tenga consciencia del cambio, es necesario cumplir con tres etapas: 1) sistema de monitoreo del entorno; 2) diseño de escenarios, y 3) selección e implementación del nuevo modelo.

El sistema de monitoreo permite a la empresa, además de identificar las señales de entorno, valorar cómo y cuánto ha cambiado el entorno en el que la organización esta inmersa, y responder las siguientes preguntas: ¿Qué modelos de negocios se están desarrollando o pueden surgir?, ¿Quién los lidera?, ¿Cómo van a afectar mi modelo de negocio y su capacidad de crear y capturar valor?. El sistema debe permitir identificar modificaciones de tipo demográfico, regulatorio y tecnológico, que permita a la empresa tratar de evaluar la magnitud del efecto de la turbulencia, así como valorar las dimensiones de dinamismo, incertidumbre y complejidad en el sector.

La segunda fase consiste en hacer una elaboración de escenarios que permitan responder a la turbulencia, buscando soluciones para hacer frente a las discontinuidades. No basta con diseñar los escenarios, es necesario construir las estrategias para cada escenario. De acuerdo con Kotler \& Caslione (2010) la construcción eficaz de escenarios requiere identificar en el entorno los generadores de la turbulencia que puedan producir caos.

Finalmente, la empresa debe seleccionar e implementar el nuevo modelo de negocios, lo que llevaría a que la empresa realice ajustes en: sistemas de producto, mercados, estructura organizativa, recursos humanos, activos, procesos. En esta etapa es necesario que exista una disposición a realizar los cambios estratégicos necesarios que han sido percibidos, ya que no todas las organizaciones son igualmente capaces de cambiar (Ginsberg, 1998; Zajac \& Shortell, 1989).
Esta modificación en el contenido de la estrategia de la empresa debe generar, en la empresa, capacidades distintivas encaminadas a tener un comportamiento creativo orientado a la innovación, una flexibilidad estratégica que permita enfrentar los momentos de dificultad de la mejor manera posible.

Para Blumenthal \& Haspeslagh (1994), el éxito del cambio estratégico implementado, no depende solo de las habilidades directivas al conducir el cambio, sino en lo acertado del diagnostico, de los aspectos estratégicos y operativos, y de si los nuevos comportamientos son los apropiados para alcanzar los objetivos de la empresa.

\section{Conclusiones}

Las organizaciones como parte de su actuar, se adaptan o se ajustan a las condiciones del entorno de manera permanente, a veces de manera natural, o como un resultado de un esfuerzo deliberado de cambio planeado. Sin embargo, en ocasiones el cambio surge como respuesta a ciertas situaciones que se presentan en el entorno, y que son involuntarios a la empresa.

Cuando al verse enfrentadas a estos acontecimientos transformadores de tipo tecnológico, regulatorio, demográficos surgen nuevos productos y servicios, se incrementa el nivel de rivalidad con los competidores, se dificultan las relaciones con proveedores y clientes, y se disminuye la capacidad de certeza al tomar decisiones, puede indicarse que el sector al que pertenece pasa por un estado de turbulencia.

La turbulencia significa siempre un aumento en el riesgo y en la incertidumbre, es una medida de aleatoriedad del estado del entorno. Aunque no se puede predeterminar con certeza el estado futuro del medio externo; si se presentan alternativas, de estados futuros, cada uno de los cuales tiene una probabilidad de ocurrencia.

Algunos pueden confundir la turbulencia del entorno con la hostilidad, o la crisis. Pero es importante aclarar que la turbulencia es un fenómeno impredecible, que se presenta por la confluencia de tres elementos: dinamismo, incertidumbre y complejidad. 
No puede ser considerado a priori como algo negativo, si bien es cierto que se generan movimientos desestabilizadores en el sector, las empresas pueden sacar provecho de la situación y llevar a cabo cambios a su interior para lograr enfrentar las discontinuidades. De esta manera lograrán tener un impacto positivo, o al menos no ver desmejorado su desempeño financiero.

Tratar de determinar el grado de turbulencia de un sector es una tara difícil, aunque existen instrumentos diversos para valorar cada una de las dimensiones (dinamismo, incertidumbre y complejidad), la información que estos suministran permite comprender el estado del entorno, pero no dar una calificación precisa sobre un nivel de turbulencia; en esto aún se trabaja; lo que se ha logrado en más de cincuenta años de reflexión alrededor del tema, es haber comprendido mejor el fenómeno, los factores que lo generen, sus consecuencias, y propuestas para enfrentarlo.

Sobre este aspecto, las alternativas van desde procesos de centralización en toma de decisiones, hasta procesos de flexibilidad estratégica. Es importante indicar que no existe una mejor alternativa para realizar el ajuste; lo que es importante es determinar si el sector presenta turbulencia, para así poder tomar alternativas graduales o radicales; dependiendo de la magnitud requerida. El dinamismo, la incertidumbre y la complejidad se constituyen en elementos que, al ser valorados, proporcionan información para tomar la decisión de qué cambiar, hacia qué cambiar y cómo causar el cambio.

En este documento se toma posición por el cambio estratégico como alternativa para enfrentar la turbulencia. Es una propuesta integradora, que le permite a la empresa aprovechar las oportunidades de la turbulencia, modificando su modelo de negocio, pero es importante que la empresa entienda el entorno y su dinámica, y reenfocar su propuesta de valor y llevarla a la práctica para recuperar posiciones.

El nuevo modelo requiere de transformaciones en políticas de corto, mediano y largo plazo, en sus activos. No son formulas simples sino que constitu- yen un conjunto de elecciones y consecuencias derivadas de esas elecciones que permitan generar o capturar valor para las empresas. La empresa debe orientarse al uso de nuevas tecnologías, sistemas y formas de distribución, procesos de innovación para atender las nuevas necesidades del cliente, de forma tal que capturar gran parte del valor creado en la nueva configuración.

Las decisiones para enfrentar la turbulencia tienen consecuencias sobre su propio funcionamiento $y$, sobre el sector en el que opera. Las consecuencias del modelo pueden ser flexibles o rígidas en función de su sensibilidad a las elecciones que las causan.

Es importante evaluar a futuro el impacto que generan los cambios implementados por las empresas en el sector, y como los actores que hacen parte de él pueden tomar medidas que amplifican el efecto de la turbulencia; llevando inclusive a ser generadores de nuevas discontinuidades. Igualmente la forma como las decisiones de la empresa pueden generar esas discontinuidades.

\section{Referencias}

Akgun, A., Byrne, J., Lynn, G., \& Keskin, H. (2007). New Product development in turbulent environments: Impact of improvisation and unlearning on new product performance. Journal of Engineering and technology Management, 24, 203-230.

Aldrich, H. (1979). Organizations and environments. Pretice Hall.

Anderson, C., Paine, F. (1975). Managerial Perceptions and Strategic Behavior The Academy of Management Journal, 18 (4), 811-823.

Andrews, K. (1980). The Concept of Corporate Strategy. Homewood, III. R.D. Irwin.

Ansoff, I. (1965). Corporate strategy: An analytic approach to business policy for growth and expansion. New York: McGrawHill.

Ansoff, Igor [1993]. El planeamiento Estratégico. Nueva tendencia de la Administración./ H Ansoff, R. P. Declerk y Hayes R. I./ Editorial Trillas, México

Ansoff, I. (1990). General management in turbulent environments. The Practising Manager, 11 (1), 2.

Ansoff, I., Eppink, J., \& Gomer, H. (1979). Management of Strategic surprise and discontinuity: problem of managerial decisiveness. Economies et Sociétés, série Sciences de Gestión (1), 459-488

Anssoff, H., \& Sullivan, P. (1993). Empirical support for a paradigmatic theory of strategic sucess behavior of enviroment serving organization. International Review of Strategic, 4, pp. 173-203. 
Argyris, C. (1985). Strategy, Change and Defensive Routines. Boston, MA: Pitman.

Ayadurai, S. \& Sohail, S. (s.f.). The Effect on Environmental Turbulence on Entrepreneurial Behaviour and Performance of Multinational Subsidiaries inMalasya. Recuperado el 27 de Agosto de 2008, de Ubinary University College: http://www.binary. edu.my/research/enviroment.pdf

Barnett, W. \& Carroll, G. (1995). Modeling Internal Organizational Change. Annual Review of Sociology, 21, 217-236.

Becherer, R. \& Maurer, J. (1998). The moderating effect of environmental variables on the entrepreneurial and marketing orientation of entrepreneur-led firms, Entrepreneurship Theory and Practice, 22(1): 47-58.

Bennis, W. (1966). Changing organizations: Essays on the development and evolution of human organization. New York: McGraw Hill.

Beesley, M. \& Hamilton, R. (1984). Small Firms' Seedbed Role and the Concept of Turbulence. The Journal of Industrial Economics, 33(2): 217-231.

Bettis, R. \& Hitt, M. (1995). The new competitive landscape. Strategic management Journal, 16, 7-19.

Bhattacharya, A., Jina J. \& Walton, A. (1996). Product-market, turbulence and time compression: three dimensions of an integrated approach to manufacturing systems design, International Journal of Operations \& Production Management, Vol. 16 No.9, pp.34-47.

Bian, D. (2006). Strategy formualtion and implementation in turbulent business enviroments: refefining organizations and their leadership formation. 202. Barcelona: IESE Business School, España.

Bitar, J. (2003). Strategy in Turbulent Environment Continuous Innovation and Generic Dynamic Capabilities. Montreal: HEC.

Blumenthal, B; Haspeslagh,P. (1994). Toward a Definition of Corporate Transformation, Sloan Management Review 35(3): 101-107

Boeker, W. (1989). Strategic change: the effects of founding and history. Academy of Management Journal, 32, 489-515.

Bourgeois, L. (1985). Strategic goals, perceived uncertainty, and economic performance in volatile environment. Academy of Management Journal , 28 (3), 548-573.

Bourgeois, L. \& Eisenhardt, K. (1988). Strategic Decision Processes in High Velocity Environments: Four Cases in the Microcomputer Industry. Management Science, 34 (7), 816-835.

Boyd, B. (1990). Corporate linkages and organizational environments: a test of resource dependence model. Strategic Management Journal, 11, 419-430.

Boyd, B.; Dess, G;. Rasheed, A. (1990) Divergence between Archival and Perceptual Measures of the Environment: Causes and Consequences. The Academy of Management Review, 18, (2),. 204-226.

Boyne, G. \& Meier, K. (2009). Environmental Turbulence, Organizational Stability, and Public Service Performance. Administration \& Society, 40 ((8)), 799-824.

Brandon, K. (2004). Change and persistence in a Christian liberal arts institution: A case study. Doctoral thesis Unpublished. Ypsilanti: Eastern Michigan University.

Brown, S. \& Eisenhardt, K. (1997). The Art of continuous change: linking Complexity theory and Time-Paced Evolution in Recentlessly Shifting Organizations. Administrative Science Quarterly, 42 ((1)), 1-34.
Brown, S. \& Eisenhardt, K. (1998). Competing on the edge: strategy as structured chaos. Boston: Harvard Business School Press.

Brown, T. \& Kirchhoff, B. (1997). The effects of resource availability and entrepreneurial orientation on firm growth, Frontiers of Entrepreneurship Research 1997. Edition. www.babson. edu/entrep/fer/papers97/kirtchoff/kir1.htm.

Buganza, T., Dell'Era, C., \& Verganti, R. (2009). Exploring the Relationship Between Product Development and Environmental Turbulence: The Case of Mobile TLC Services. Product Innovation Managament, 26, 308-321.

Burns, T. \& Stalker, G. (1961). The Management of Innovation. London: Tavistock.

Calantone, R., Garcia, R. \& Droge, C. (2003). The Effects of Environmental Turbulence on New product Development Strategy Planning. Journal of product Innovation Management, 20, 90-103.

Calantone, R., Schmidt, J. \& Di Benedetto, A. (1997). New product Activities and Performance: The Moderating Role of Environmental Hostility. Journal of Product Innovation Management, 14, 179-189.

Caldart, A. \& Ricart, J. (2006). Corporate Strategy in turbulent Environments: Key Roles of the Corporate Level. Working Paper (623), 31. Barcelona, Spain: IESE Business School University of Navarra.

Callot, P. (1997), Déterminants Structurels et Stratégiques, Perception de l'Environnement et Influence sur le Champ Concurrentiel de la Restauration Commerciale en France. Thèse, IAE de Poitiers, 6 janvier 1997, 482 p.

Cameron, K. (1984). Organizational Adaptation and Higher Education. The Journal of Higher Education, 55 (2), 122-144.

Cameron, K., Kim, M. \& Whetten, D. (1987). Organizational Effects of Decline and Turbulence. Administrative Science Quarterly, 32, 222-240.

Camillus, J. \& Datta, D. (1991). Managing strategic issues in a turbulent environment. Long Range Planning, 24 (2), 67-74.

Castrogiovanni, G. J. (1991). Environmental munificence: A theoretical assessment. Academy of Management Review, 16 (3), 542-565.

Chaffee, F. (1985). Three Models of Strategy. Academy of Management Review, 10 (1), 89-98.

Chakravarthy, B. (1997). A New Strategy Framework for Coping with Turbulence. Sloan Management Review, 69-82.

Child, J. (1975). Managerial and Organizational Factors Associated with Company Performance, part II- A Contingency Analysis. Journal of Management Studies, 12 (1-2), 12-27.

Child, J. (1972). Organizational Structure, Environment and Performance: The Role of Strategic Choice. Sociology, 6 (1), 122

Coutney, H. (2001). 20/20 foresight: crafting strategy in an uncertain world Boston, Mass. Harvard Business School Press.

Covin, J. \& Slevin, D. (1989). Strategic Management of Small Fir$\mathrm{ms}$ in Hostile and Benign Environments. Strategic Management Journal, 10 (1), 75-87.

Craig, T. (1996). The Japanese beer Wars: Initiating and Responding to Hypercompetition in New Product Development. Organization Science, 7 (3), 302-321.

Crossan, M., Vera, D.m \& Nanjad, L. (2008). Transcendent leadership: Strategic leadership in dynamic environments. The leadership quarterly, 19 ((5)), 569-581. 
D'Aveni, R. (2004). The rise of Hypercompetition from 1950 to 2002: Evidence of increasing structural destabilization and temporary competitive advantage.

D'Aveni, R. (1994). Hypercompetition. Managing the Dynamics of Strategic Manauvering. New York: Free Press.

Daft, R., Sormusen,J. \& Parks, D. (1988). Chief executive scanning, environmental characteristics and company performance: an empirical study. Strategic Management Journal, 9, 123-139.

Dante, R. (2002). Estrategia de Ventas en Tiempos de Turbulencia. Recuperado el 10 de Agosto de 2008, de http://fce.uncu. edu.ar/investigacion/Jornadas/jornadas2002/Dis/Administracion/54/T54.pdf

Davis, D., Morris, M. \& Allen, J. (1991). Perceived environmental turbulence and its effects on selected entrepreneurship, marketing and organizational characteristics in industrial firms. Journal of the Academy of Marketing Science, 19 (1), 43-51.

Dess \& Beard, D. (1984). Dimensions of organizational task environments. Administrative Science Quarterly, 52-73.

Dess, G., Lumpkin, G. \& Covin, J. (1997). Entrepreneurial strategy making and firm performance: tests of contingency and configurational models. Strategic Management Journal, vol. 18(9): 677-695.

Dessler, G. (1992). Organization theory: Integrating structure and behaviour (Second edition ed.). London,UK: Prentice-Hall International Limited.

Downey, H. H. (1975). Environmental uncertainty: the construct and its application. Administrative Science Quarterly, 20, 613629.

Downey, H., Slocum, J. (1975). Uncertainty: Measures, Research, and Sources of Variation. The Academy of Management Journal, Vol. 18, (3): 562-578

Droge, C., Calantone, R. \& Harmancioglu, N. (2008). New Product Success: Is It Really Controllable by Managers in Highly Turbulent Environments? The Journal of Product Innovation Management (25), 272-286.

Drucker, P. (1980). Managing in Turbulent Times. New York: Harper \& Row.

Dufour, Y. \& Steane, P. (2006). Competitive paradigms on strategic change: mapping the field and further research development. Strategic Change, 15 (3), 129-144.

Duncan, R. (1972). Characteristics of Organizational Environments and perceived Environmental Uncertainty. Administrative Science Quarterly, 17 (3).

Duncan, R. (1974). Multiple Decision-making Structures in Adapting to Environmental Uncertainty: The Impact on Organizational Effectiveness. Human Relations, 26 (3), 273-291.

Dunphy, D. (1996). Organizational Change in Corporate Settings. Human Relations 49: 541-552.

Dutton, J. \& Duncan,R. (1987). Strategic Issue Diagnosis and Creation of Momentum for Change. Strategic Management Journal, 8 (3), 279-295.

Dwyer, R. \& Welsh, A. (1985). Environmental Relationship of the Internal Political Economy of Marketing Channels. Journal of Marketing Research, XII, 397-414.

El Sawy, O. \& Pavlou, P. (2008). IT-Enabled Business capabilities for Turbulent Environments. MIS Quarterly Executive, 7 (3), 139-150.

Emery, F. \& Trist, E. (1965). The Causal Texture of Organizational Environments. Human Relations, 18 (21), 21-32.
Fiss, P., \& Zajac, E. (2006). The symbolic management of strategic change: sense - giving via framing and decoupling. Academy Management , 49, 1173-1193.

Forbes, D., Manrakhan, S. \& Banerjee, S. (2004). Strategic responses to an environmental jolt: Executive turnover in Internet IPOs. The Journal of Private Equity, 8, 63-93.

Freeman, C. \& Soete, L. (1997). The economics of industrial Organization. MIT. 1997. Cambridge. US.: MIT.

Galbraith, J. (1973). Designing Complex Organizations. Reading, MA: Addison-Wesley Publishing Co.

Ganesan, S. (1994). Determinants of Long-Term Orientation in Buyer-Seller Relationships. The Journal of Marketing, 58 (2), 1-19.

García, S., Dolan, S. \& Navarro, C. (1999). La dirección por valores para animar la empresa en entornos turbulentos. Harvard Deusto business review, 78-89.

Ginsberg, A. (1998). Measuring and modelling changes in strategy: Theoretical foundations and empirical directions. Strategic Management Journal, 9: 559-575.

Gioia, D. A. \& Chittipeddi, K. (1994). Sense making and sense giving in strategic change initiation. Strategic Management Journal, 12, 433-448.

Gioia, D., Thomas, J. J., Clark, S., \& Chittipeddi, K. (1994). Symbolism and strategic change in academia: The dynamics of sense making and influence. Organizational Science, 5, 363-383.

Glazer, R. \& Weiss, A. (1993). Marketing in Turbulent Environments: Decision Processes and the Time-Sensitivity of Information. Journal of Marketing Research, 30 (November), p. 509-521.

Gordon, S.S., Stewart, W.H., Sweo, R., \& Luker, W.A. (2000). Convergence versus strategic reorientation: The antecedents of fast-paced organizational change, Journal of Management, 26, 911-945.

Gray, B. \& Ariss, S. (1985). Politics and strategic change across organizational life cycles. Academy of Management Review, 10 (4), 707-723

Grant, R. (2003). Strategic Planning in a Turbulent Environment: Evidence from Oil Majors. Strategic Management Journal, 24, 491-517.

Greenspan, A. (2007). The Age of Turbulence: Adventures in a New World. Nueva York: Penguin.

Gueguen, G. (2001). Http://Www.Sciencesdegestion.Com/These/These.Zip.

Gueguen, G. (1997). Face aux turbulences, l'entreprise doit-elle être réactive? Propos de réflexion. Vlème conférence de I'AIMS. Montreal: HEC.

Hall, R. (1996). Organizaciones, Estructuras, Procesos, y Resultados. México: Prentice Hall.

Hall, J. \& Rosson, P. (2006). The Impact of technological Turbulence on Entrepreneurial behavior, Social Norms and Ethics: Three Internet-based Cases. Journal of Business Ethics , 64, 231-248.

Hambrick, D. (1983). High Profit Strategies in Mature Capital Goods Industries: A Contingency Approach. The Academy of Management Journal , 26 (4), 687-707

Head, T. (2005). Structural Changes in turbulent Environments: A Study of Small and Mid-Size Chinese Organizations. Journal of Leadership \& Organizational Studies, 12 (2), 82-93.

Hofer, C. \& Schendel, D. (1978). Strategy formulation: Analytical concepts. St. Paul: West Pub. Co. (St. Paul). 
Huber, G. (1984). The nature and design of post-industrial environments, Management Science, 30: 928-951.

Ireland, R., Hitt, M., Bettis, R. \& Porras, D. (1987). Strategy formulation processes: differences in perceptions of strength and weakness indicators and environmental uncertainty by managerial level, Strategic Management Journal, 8: 469-485.

Janis, I. (1962). Psychological effects of warnings. In G. W. Baker and D. W. Chapman (eds,), Man and Society in Disaster: 5692, New York: Basic Books,

Jaworski, B. \& Kohli, A. (1993). Market Orientation: Antecedents and Consequences. Journal of Marketing , 57 (3), 53-70.

Jennings, D., \& Seaman, S. (1994). High and low levels of organizational adaptation: an empirical analysis of strategy, structure and performance. Strategic Management Journal, 15, 459-475.

Joffre, P. \& Koenig, G. (1984). Stratégies de coopération et d'alliance interentreprises. Enseignement et Gestion, $n^{\circ} 31$, otoño.

Johnson, G. \& Scholes, K. (1999). Exploring Corporate Strategy: Text and Cases. Harlow, England: Prentice Hall.

Jurkovich, R. (1974). Core Typology of Organizational Environments. Administrative Science Quarterly, 19 (3).

Kalika, M. (1985). L'efficacité des entreprises est-elle liée à leur structure ? Revue Française de Gestion , 93-104.

Khandawalla, P. (1972). Environment and its impact on the organization. International Studies of Management and Organization, 2, 297-313.

Khandwalla, P. (1974). Mass Output Orientation of Operations Technology and Organizational Structure. Administrative Science Quarterly, 19 (1), 74-97.

Khandwalla, P. (1976-1977). Some top Management styles, their context and performance. Organizations and Administrative Sciences, 7 (4), 21-51.

Kotler, P., \& Caslione, J. (2010). Caótica. Bogotá: Grupo Editorial Norma.

Kraatz, M. \& Zajac, E. (2001). How Organizational Resources Affect Strategic Change and Performance in Turbulent Environments: Theory and Evidence. Organization Science, 12 (5), 632-657.

Kuivalainen, O., Sundqvist, S., Puumalainen, K. \& Cadogan, J. (2004). The Effect of Environmental Turbulence and Leader Characteristics on International Performance: Are Knowledge-Based Firms Different? Canadian Journal of Administrative Sciences, 21 (1), 35-50

Langley, A. \& Denis, J. (2006). Neglected Dimensions of Organizational Change: Towards. En R. Lines, I. Stensaker \& A. Langley, \& A. Langley, New Perspectives on Organizational Change and Learning (págs. 136-159).

Lawless, M. \& Finch,K. (1989). Choice and Determinism: a Test of Hrebiniak and Joyce's Framework on Strategy - Environment Fit, Strategic Management Journ a I, 10, 351-365.

Lawrence, P. R. \& Lorch, J. W. (1967). Organization and Environment. Homewood: Irwin.

Leker, J. (2001). Reorientation in a Competitive Environment: An Analysis of Strategic Change. Schmalenbach Business Review , 53, 41-55

Lewin, K. (1951). Field theory in social science; selected theoretical papers. New York: Harper \& Row.
Lewin, A. Y., \& Volberda, H. W. (1999). Prolegomena on coevolution: A framework for research on strategy and new organizational forms. Organization Science, 10: 519-534.

Li, H. \& Atuahene-Gima, K. (2001). The Impact of Interaction between R\&D and Marketing on New Product Performance: An Empirical Analysis of Chinese High Technology Firms. International Journal of Technology Management , 21 ((1/2)), 61-75.

Lichtenthaler, U. (2009). Absotive Capacity, Environmental Turbulence, and The Complementarity of Organizational Learning Processes. Academy of Management Journal, 52 (4), 822-846.

Lin, X. \& Germain, R. (2003). Organizational Structure, Context, Customer Orientation, and Performance: Lessons from Chinese State-Owned Enterprises. Strategic Management Journal, Vol. 24, No. 11: 1131-1151.

Lindsay, W. \& Rue, L. W. (1980). Impact of the business environment on long range planning process: A contingency view. Academy of Management Journal, 23: 385-404.

Lorsch, J.W. (1986). "Managing Culture: The Invisible Barrier to Strategic Change". CaliforniaManagementReview, 28:95-109.

Makridakis, S. (1979). Accuracy of Forecasting: An Empirical Investigation. Journal of the Royal Statistical Society. Series A (General), 142, 2. 97-145.

Markoczy, L. (2000). La innovación estratégica en empresas ya establecidas. Harvard Deusto Business Review (81), 22-32.

Mason, R. (2006). Coping with compelxity and turbulence- An entrepreneurial solution. Journal of Enterpresing Culture , 14 (4), 241-266.

McCann, J. \& Selsky, J. (1984). Hyperturbulence and the Emergence of Type 5 Environments. Academy of Management Review, 9 (3), 460-470.

Metcalfe, J. (1974). Systems Models, economics Models and the Causal Texture of Organizational Environments: An Approach to Macro-Organization Theory. Human Relations , 27 (7), 639-663.

Metcalfe, L., \& McQuillan, W. (1977). Managing Turbulence. TIMS/Studies in the Management Science, 5, 7-23.

Menninger, W. C. (1952). Psychological reactions in an emergency flood, American Journal of Psychiatry, 109: 128-130.

Meyer, A. (1982). Adapting to Environmental Jolts. Administrative Science Quarterly, 27, 515-537.

Miles, R. \& Snow, C. (1978). Organizational Strategy, Structure and Process. McGraw-Hill.

Miller, D. (1982). Evolution and revolution: a quantum view of structural change in organizations. The Journal of Management Studies , 19 (2), 131-152.

Miller, D. (1988). Relating Porter's business strategies to environment and structure: analysis and performance implications. Academy of Management Journal, 31 (2), 280-308.

Miller, D. (1983). The correlates of entrepreneurship in three types of firms. Management Science, 29 (7), 770-791.

Miller, D. \& Droge, C. (1986). Psychological and traditional determinants of structure. Administrative Science Quarterly, 31, 539-560.

Miller, D. \& Friesen, P. (1982). Innovation in conservative and entrepreneurial firms: two models of strategic momentum. Strategic Management Journal, 3, 1-25.

Milliken, F. (1990). Perceiving and interpreting environmental change: an examination of college administrators interpreta- 
tion of changing demographics". Academy of Management Journal , 33 (1), 42-63.

Milliken, F. (1987). Three Types of perceived Uncertainty About the Environment: State, Efefct, and Response Uncertainty. Academy of Management Review, 12 (1), 133-143.

Mintzberg, H. (1990). The design school: Reconsidering the basic prernises of strategic management, Strategic Management Journal, 11, 171-195.

Mintzberg, H., Ahlstrand, B. \& Lampel, J. (1988). Strategy Safari: A Guided Tour Through the Wilds of Strategic Management. New York: The Free Press.

Mintzberg, H. (1994). Rethinking Strategic Planning Part I. Pitfalls and Fallacies. Long range planning. 27 (3);12-21.

Morris, M., Hansen \& Pitt (1995). Environmental turbulence and organizational buying: the case of health benefits in south Africa. Industrial Marketing Management (24), 305-315.

Mullins, J. Y Cummings, L. (1999). Situational strength. A framework for understanding the role of individuals in initiating proactive strategic change. Journal of Organizational Change Management, 12: 462-479.

Nadler, D. \& Tushman, M. (1989). Organizational frame bending: principles for managing reorientation. Academy of Management Executive, 3 (3), 194-204.

Nag, R., Hambrick, D. \& Chen, M. (2007). What is Strategic Management Really Inductive Derivation of a Consensus Definition of The Field. Strategic Management Journal, 935-955.

Naman, J. \& Slevin, D. (1993). Entrepreneurship and the concept of fit: a model and empirical tests. Strategic Management Journal, 14, 137-153.

Narasimha, S. (2001). Strategy in turbulent Environments: The Role of Dynamic Competence. Managerial and decision Economics (22), 201-212.

Pascale, R. (1999). Surfing the edge of chaos. Sloan management review, 40 (3), 83-95.

Pettus, M., Kor, Y., \& Mahoney, J. (2007). A Theory of Change in Turbulent Environments: The Sequencing of Dynamics Capabilities Following Industry Deregulation. The International Journal of Strategic Change Management , 1 (3), 186-211.

Pfeffer, J. \& Salancik, G. (1978). The external control of organizations. New York.: Harper \& Row.

Pine II, J. (1993). Mass customization: the new frontier in business competition. Boston: Harvard Business School Press.

Polley, D. (1997). Turbulence in Organizations: New Metaphors for Organizational research. Organization Science, 8 (5), 445-457.

Porter, M. (1991). Towards a dinamic theory of strategy. Strategic Management Journal , 12, 95-117.

Post, J. \& Mahon, J. (1980). Articulated turbulence: The effect of regulatory agencies on corporate responses to social change. Academy of Management Review, 5 (3), 399.

Power, B. \& Reid, G. (2005). Flexibility, Firm-Specific Turbulence and the Performance of the Long-lived Small Firm. Review of Industrial Organization, 26 (4), 415-443.

Prokresch, S. (1993). Mastering chaos at the high-tech Frontier: an interview with Silicon Graphic's ed McCracken. Harvard business review, 71 (6), 134-144.

Quinn, J. (Winter de 1980). An incremental approach to strategic change. The Mckinsey Quarterly, 34-52.
Radovic, M. (2008). Managing the Organizational Change and Culture in the Age of Globalization. Journal of Business Economic and Management (1), 3-11.

Rajagopalan, N. \& Spreitzer, G. (1997). Toward a theory of strategic change: a multi-lens perspective and integrative framework. Academy of Management Review, 22 (11), 48-79.

Ramos-Rodríguez, A. \& Ruíz-Navarro, J. (2004). Changes in the intellectual structure of strategic management research: a bibliometric study of the Strategic Management Journal, 19802000. Strategic Management Journal, 25 (10), 981-1004.

Reimann, B. \& Wiener, Y. (1986). Corporate culture and strategy change: analyzing the source and focus of shared values. Academy of Management. Chicago.

Ricart, J. (2010). El modelo de negocio motor del cambio sectorial. IESE insight (4), 13-20.

Rigby, D. (2001). Winning in Turbulence. London. Perseus Distribution Services

Sadler, P. (1996). Managing Change. London: kogan Page.

Sawyerr, O. (1993). Environmental uncertainty and environmental scanning activities of Nigerian manufacturing executives: a comparative analysis. Strategic Management Journal, 14, 287-299.

Siggelkow, N . \& Rivkin, J. (2005). Speed and Search: Designing Organizations for Turbulence and Complexity. Organization Science, 16, 101-122.

Silverblatt, R. \& Korgaonkar, P. (1987). Strategic Market Planning in a Turbulent Business Environment. Journal of Business Research, 15, 339-358

Slater, S. \& Narver, J. (1994). Does competitive environment moderate the market orientation-performance relationship? Journal of Marketing, 58: 46-55.

Sosa, S. (2003). La Génesis y el desarrollo del cambio estratégico: Un enfoque dinámico basado en el momentum organizativo. Tesis Doctoral no publicada. Las Palmas de Gran Canaria: Universidad de las Palmas de Gran Canaria.

Souba, W. (2003). The New Leader: New Demands in a Changing Turbulent Environment. The American College of Surgeon, 79-87.

Souder, W., Sherman, D. \& Davies-Cooper, R. (1998). Environmental Uncertainty, Organizational Integration, and New Product Development Effectiveness: A Test of Contingency Theory. Journal of Product Innovation Management (15), 520533.

Souder, W and Song, M. (1998), Analyses of U.S. and Japanese management Processes Associated with New Product Success and Failure in High and Low Familiarity Markets, The Journal of Product Innovation Management, 15 (3), 208-223.

Stacey, R. (1995), The science of complexity: an alternative perspective for strategic change processes. Strategic Management Journal, 16. 477-495

Starbuck, W., Greve, A. \& Hedberg, B. (1978). Responding to crisis, Journal of Business Administration. 9:111-137.

Staw, B. \& Szwajkowski, E. (1975). The scarcity-munificence component of organizational environmentsand the commision of illegal acts. Administrative Science quarterly, 20, 34535.

Steiner, G. \& Miner, J. (1985). Management policy and strategy: Text, readings, and cases. Nueva York, Macmillan. New York: Macmillan. 
Stigter, H. (2002). Co-operation as a response to a turbulent environment. Zoetermer: EIM.

Suikki, R., Tromstedt, R. \& Haapasalo, H. (2006). Project management competence development framework in turbulent business environment. Technovation, 26, 723-738.

Sull, D. (2009). The Upside of turbulence. New York: Harper Collins Publisher.

Sutcliffe, K. \& Huber, G. (1998). Firm and industry as determinants of executive perceptions of the environment. Strategic Management Journal, 793-807.

Tamayo, I, Gutiérrez, L; Martínez, F (2007). La percepcion de la necesidad del cambio estrategico como fuente de ventaja competitiva: una aproximacion teórica. En: El comportamiento de la empresa ante entornos dinámicos: XIX Congreso anual y XV Congreso Hispano Francés de AEDEM, Vol. 1, 2007 (Ponencias), pag. 38.

Tan, J., Li, S. \& Li, W. (2006). Building Core Competences in a Turbulent Environment: An Exploratory Study of Firm Resources and Capabilities in Chinese Transitional Economy. Managing Global Transitions, 4 (3), 197-214.

Tetenbaum, T. (1998). To Plan or Not to Plan, Management Review , 87 (9), 70.

Terreberry, S. (1968). The Evolution of Organizational environments. Administrative Science Quarterly , 12, 590-613.

Thompson, J. (1967). The organizational environment and how it is known. En J. Thompson, Organizations in Action (págs. 62-91). New York: Mc Graw Hill.

Tichy, N. (1982). Managing change strategically: the technical, political and cultural keys. Organizational Dynamics, 11 (2), 59-80.

Tosi, H., Aldag, R. \& Storey, R. (1973). On the measurement of the environment. Administrative Science Quarterly. 18: 2736.

Trist, E. (1980). The environment and system-response capability. Futures, 12 (4), 113-127.

Tushman, M. \& Anderson, P. (1986). Technological Discontinuities and Organizational Environments. Administrative Science Quarterly, 31 (3), 439-465.
Ulrich, D. \& Wiersema, M. (1989). Gaining Strategic and Organizational Capability in a Turbulent Business Environment. The Academy of management Executive, III (2), 115-122.

Van De Ven A. H \& Poole M. S. (1995), Explaining Development and Change in Organizations", Academy of Management Review, 20, (3), 510-540.

Venkataraman, S., Van de Ven, A., Buckeye, J. \& Hudson, R. (1990). Startig Up in a Turbulent Environment: A Process Model of failure Among Firms With High Customer Dependence. Journal of Business Venturing, 5, 277-295.

Virany, B., Tushman, M. \& Romanelli, E. (1992). Executives Succesion and orgaqnization Outcomes in Turbulent Environments: An Organization Learning Approach. Organization Science, 3 (1), 72-91.

Volberda, H. (1997). Building Flexible Organizations for Fast-moving Markets. Long Range Planning, 30 (2), 169-183.

Waterhouse, M. (1992). Managing effectively in turbulent environments. Journal of Strategic Change, 1, 135-146.

Watzlawick, P; Weakland, J; Fish, R. (1974). Change. Principles of problem Formation and Resolution. New York: Norton and Co.

Weick, K. \& Quinn, J. (1999). Organizational Change and development. Annual review of Psichology, 50, 361-386.

Withey, S. B. (1962). Reaction to uncertain threat. In G. W, Baker and D. W. Chapman (eds, \}. Man and Society in Disaster: 93-123, New York: Basic Books.

Woodward, S. (1982). The myth of turbulence. Futures, 14 (4), 266-279.

Zahra, A. \& George, G. (2002). Absorptive Capacity: A Review, Reconceptualization, and Extension. The Academy of Management Review, 27 (2), 185-203.

Zajac, E; Shortell, S. (1989). Changing Generic Strategies: Likelihood, Direction, and Performance Implications, Strategic Management Journal, 10:413-430.

Zajonc, R. (1965). Social facilitation. Science, 149: 269-274.

Zamutto, R. \& Cameron, K. (1985). Environmental Decline and Organizational Response. Researching Organizational Behavior, 223-262. 